\title{
WEIGHT ANNOTATION IN INFORMATION EXTRACTION *
}

\author{
JOHANNES DOLESCHAL $^{a}$, BENNY KIMELFELD $^{b}$, WIM MARTENS $^{c}$, AND LIAT PETERFREUND $^{d}$ \\ ${ }^{a}$ University of Bayreuth, Germany; Hasselt University, Belgium \\ e-mail address: johannes.doleschal@uni-bayreuth.de \\ ${ }^{b}$ Technion, Haifa, Israel \\ e-mail address: bennyk@cs.technion.ac.il \\ ${ }^{c}$ University of Bayreuth, Germany \\ e-mail address: wim.martens@uni-bayreuth.de \\ ${ }^{d}$ DI-ENS, ENS, CNRS, PSL University, and Inria, Paris, France \\ e-mail address: liatpf.cs@gmail.com
}

\begin{abstract}
The framework of document spanners abstracts the task of information extraction from text as a function that maps every document (a string) into a relation over the document's spans (intervals identified by their start and end indices). For instance, the regular spanners are the closure under the Relational Algebra (RA) of the regular expressions with capture variables, and the expressive power of the regular spanners is precisely captured by the class of VSet-automata - a restricted class of transducers that mark the endpoints of selected spans.

In this work, we embark on the investigation of document spanners that can annotate extractions with auxiliary information such as confidence, support, and confidentiality measures. To this end, we adopt the abstraction of provenance semirings by Green et al., where tuples of a relation are annotated with the elements of a commutative semiring, and where the annotation propagates through the positive RA operators via the semiring operators. Hence, the proposed spanner extension, referred to as an annotator, maps every string into an annotated relation over the spans. As a specific instantiation, we explore weighted VSet-automata that, similarly to weighted automata and transducers, attach semiring elements to transitions. We investigate key aspects of expressiveness, such as the closure under the positive RA, and key aspects of computational complexity, such as the enumeration of annotated answers and their ranked enumeration in the case of ordered semirings. For a number of these problems, fundamental properties of the underlying semiring, such as positivity, are crucial for establishing tractability.
\end{abstract}

Key words and phrases: Information extraction, regular document spanners, weighted automata, provenance semirings, K-relations.

* An abridged version of this article has been published in the 2020 International Conference on Database Theory [DKMP20].

니밈

LCOGICAL METHODS N COMPUTER SCIENCE
DOI:10.46298/LMCS-18(1:21)2022

(c) J. Doleschal, B. Kimelfeld, W. Martens, and L. Peterfreund

(c) Creative Commons 


\section{INTRODUCTION}

A plethora of paradigms have been developed over the past decades towards the challenge of extracting structured information from text - a task generally referred to as Information Extraction (IE). Common textual sources include natural language from a variety of sources such as scientific publications, customer input and social media, as well as machine-generated activity logs. Instantiations of IE are central components in text analytics and include tasks such as segmentation, named-entity recognition, relation extraction, and coreference resolution [Sar08]. Rules and rule systems have consistently been key components in such paradigms, yet their roles have varied and evolved over time. Systems such as Xlog [SDNR07] and SystemT $\left[\mathrm{CKL}^{+} 10\right]$ use IE rules for materializing relations inside relational query languages. Machine-learning classifiers and probabilistic graphical models (e.g., Conditional Random Fields) use rules for feature generation [LBC04, SM12]. Rules serve as weak constraints (later translated into probabilistic graphical models) in Markov Logic Networks [PD07] (abbrev. MLNs) and in the DeepDive system [SWW $\left.{ }^{+} 15\right]$. Rules are also used for generating noisy training data ("labeling functions") in the Snorkel system $\left[\mathrm{RBE}^{+} 17\right]$.

The framework of document spanners (spanners for short) provides a theoretical basis for investigating the principles of relational rule systems for IE [FKRV15]. Specifically, a spanner extracts from a document a relation over text intervals, called spans, using either atomic extractors or a relational query on top of the atomic extractors. More formally, by a document we refer to a string $d$ over a finite alphabet, a span of $d$ represents a substring of $d$ by its start and end positions, and a spanner is a function that maps every document $d$ into a relation over the spans of $d$. The most studied spanner language is that of the regular spanners: atomic extraction is via regex formulas, which are regular expressions with capture variables, and relational manipulation is via the relational algebra: projection, natural join, union, and difference. Equivalently, the regular spanners are the ones expressible as variable-set automata (VSet-automata for short), which are nondeterministic finite-state automata that can open and close variables (playing the role of the attributes of the extracted relation). Interestingly, there has been an independent recent effort to express artificial neural networks for natural language processing by means of finite-state automata [WGY18, MY18, $\mathrm{MSV}^{+} 19$ ].

To date, the research on spanners has focused on their expressive power [FKRV15, PtCFK19, Fre19, SS21], the computational complexity of their main algorithmic problems [ACJR19, ABMN19, FKP18, FRU ${ }^{+}$18], incompleteness [MRV18, PFKK19], and other system aspects such as cleaning [FKRV16] and distributed query planning [DKM $\left.{ }^{+} 19\right]$. That research has exclusively adopted a Boolean approach: a tuple is either extracted or not. Nevertheless, when applied to noisy or fuzzy domains such as natural language, modern approaches in artificial intelligence adopt a quantitative approach where each extracted tuple is associated with a level of confidence that the tuple coincides with the intent. When used within an end-to-end IE system, such confidence can be used as a principled way of tuning the balance between precision and recall. For instance, in probabilistic IE models (e.g., Conditional Random Fields), each extraction has an associated probability. In systems of weak constraints (e.g., MLN), every rule has a numerical weight, and the confidence in an extraction is an aggregation of the weights of the invoked rules that lead to the extraction. IE via artificial neural networks typically involves thresholding over a produced score or confidence value $\left[\mathrm{CN} 16, \mathrm{PPQ}^{+} 17\right]$. Numerical scores in the extraction process 
are also used for quantifying the similarity between associated substrings, as done with sequence alignment and edit distance in the analysis of biological sequences such as DNA and RNA [TSZB03, WJ94].

In this work, we embark on the investigation of spanners that quantify the extracted tuples. We do so by adopting the concept of annotated relations from the framework of provenance semirings by Green et al. [GKT07]. In essence, every tuple of the database is annotated with an element of a commutative semiring, and the positive relational algebra manipulates both the tuples and their annotations by translating relational operators into semiring operators (e.g., product for natural join and sum for union). An annotated relation is referred to as a $\mathbb{K}$-relation, where $\mathbb{K}$ is the domain of the semiring. The conceptual extension of the spanner model is as follows. Instead of a spanner, which is a function that maps every document $d$ into a relation over the spans of $d$, we now consider a function that maps every document $d$ into a $\mathbb{K}$-relation over the spans of $d$. We refer to such a function as a $\mathbb{K}$-annotator. Interestingly, as in the relational case, we can vary the meaning of the annotation by varying the semiring.

- We can model confidence via the probability (a.k.a. inside) semiring and the Viterbi (best derivation) semiring [Goo99].

- We can model support (i.e., number of derivations) via the counting semiring [Goo99].

- We can model access control via the semiring of the confidentiality policies [FGT08] (e.g., does the extracted tuple require reading top-secret sections? which level suffices for the tuple?).

- We can model the traditional spanners via the Boolean semiring.

As a specific instantiation of $\mathbb{K}$-annotators, we study the class of $\mathbb{K}$-weighted VSetautomata. Such automata generalize VSet-automata in the same manner as weighted automata and weighted transducers (cf., e.g., the Handbook of Weighted Automata [DKV09]): transitions are weighted by semiring elements, the cost of a run is the product of the weights along the run, and the weight (annotation) of a tuple is the sum of costs of all the runs that produce the tuple. (Again, there has been recent research that studies the connection between models of artificial neural networks in natural language processing and weighted automata [STS18].)

Our investigation answers several fundamental questions about the class of $\mathbb{K}$-weighted VSet-automata:

(1) Is this class closed under the positive relational algebra (according to the semantics of provenance semirings [GKT07])?

(2) What is the complexity of computing the annotation of a tuple?

(3) Can we enumerate the annotated tuples as efficiently as we can do so for ordinary VSet-automata (i.e., regular document spanners)?

(4) In the case of ordered semirings, what is the complexity of enumerating the answers in ranked order by decreasing weight?

Our answers are mostly positive and show that $\mathbb{K}$-weighted VSet-automata possess appropriate expressivity and tractability properties. As for the last question, we show that ranked enumeration is intractable and inapproximable for some of the aforementioned semirings (e.g., the probability and counting semirings), but tractable for positively ordered and bipotent semirings, such as the Viterbi semiring. 
Comparison to the conference version. This article is an extended version of [DKMP20], which was published at the 23rd International Conference on Database Theory. In addition to the full proofs, we implemented the following changes:

(1) We define a more precise cost model (Section 5) and use it throughout the entire article for complexity analysis. The cost model in [DKMP20] used unit-cost for all semiring operations, which is sometimes unrealistic. The model we adopt is more precise in the sense that we encode semiring elements in binary. We define what it means for a semiring to have an efficient encoding, which essentially is the case when linear sequences of semiring operations can be performed in polynomial time. Furthermore, we show that the semirings that we are interested in our article indeed have efficient encodings.

(2) We extended a result on closure properties (Section 6), namely that joins preserve unambiguity (Lemma 6.9).

(3) We present additional characterizations of recognizable $k$-ary string relations in terms of selectability with $\mathbb{K}$-annotators. We proved a characterization in [DKMP20] in terms of positive semirings $\mathbb{K}$ (Theorem 6.11). We show in Section 6.4.1 the result can be extended beyond positive semirings. We identify a number of properties on the semiring that are sufficient for the equivalence to hold and show that the equivalence also holds for the Lukasiewcz semiring, which is not positive.

Organization. The remainder of this article is organized as follows. We give some required algebraic background, preliminary definitions and notation in Section 2. In Sections 3 and 4 we define $\mathbb{K}$-Annotators and weighted VSet-automata - a formalism to represent $\mathbb{K}$-Annotators. We study their fundamental properties in Section 6 and various evaluation and enumeration problems of weighted VSet-automata in Sections 7, and 8. We conclude in Section 9 .

\section{Preliminaries}

Weight annotators read documents and produce annotated relations [GKT07], which are relations in which each tuple is annotated with an element from a commutative semiring. In this section, we revisit the basic definitions and properties of annotated relations.

2.1. Algebraic Foundations. We begin by giving some required background on algebraic structures like monoids and semirings [Gol99].

A commutative monoid $(\mathbb{M}, *$, id) is an algebraic structure consisting of a set $\mathbb{M}$, a binary operation $*$ and an element id $\in \mathbb{M}$, such that:

(1) $*$ is associative, i.e., $(a * b) * c=a *(b * c)$ for all $a, b, c \in \mathbb{M}$,

(2) $*$ is commutative, i.e. $a * b=b * a$ for all $a, b \in \mathbb{M}$, and

(3) id is an identity, i.e., id $* a=a$ for all $a \in \mathbb{M}$.

We say that a monoid ( $\mathbb{M}, *$, id) is bipotent, if $a * b \in\{a, b\}$, for every $a, b \in \mathbb{M}$.

A commutative semiring $(\mathbb{K}, \oplus, \otimes, \overline{0}, \overline{1})$ is an algebraic structure consisting of a set $\mathbb{K}$, containing two elements: the zero element $\overline{0}$ and the one element $\overline{1}$. Furthermore, it is equipped with two binary operations, namely addition $\oplus$ and multiplication $\otimes$ such that:

(1) $(\mathbb{K}, \oplus, \overline{0})$ and $(\mathbb{K}, \otimes, \overline{1})$ are a commutative monoids,

(2) multiplication distributes over addition, that is, $(a \oplus b) \otimes c=(a \otimes c) \oplus(b \otimes c)$ for all $a, b, c \in \mathbb{K}$, and 
(3) $\overline{0}$ is absorbing for $\otimes$, that is, $\overline{0} \otimes a=\overline{0}$ for all $a \in \mathbb{K}$.

Furthermore, a semiring is positive if, for all $a, b \in \mathbb{K}$, the following conditions hold:

- $\overline{0} \neq \overline{1}$,

- if $a \oplus b=\overline{0}$, then $a=\overline{0}=b$, and

- if $a \otimes b=\overline{0}$, then $a=\overline{0}$ or $b=\overline{0}$.

We call a semiring bipotent, if its additative monoid is bipotent.

An element $a \in \mathbb{K}$ is a zero divisor if $a \neq \overline{0}$ and there is an element $b \in \mathbb{K}$ with $b \neq \overline{0}$ and $a \otimes b=\overline{0}$. Furthermore, an element $a \in \mathbb{K}$ has an additive inverse, if there is an element $b \in \mathbb{K}$ such that $a \oplus b=\overline{0}$. In the following, we will also identify a semiring by its domain $\mathbb{K}$ if the rest is clear from the context. When we do this for numeric semirings such as $\mathbb{Q}$ and $\mathbb{N}$, we always assume the usual addition and multiplication.

Given a semiring $(\mathbb{K}, \oplus, \otimes, \overline{0}, \overline{1})$ and a set $\mathbb{K}^{\prime} \subseteq \mathbb{K}$ with $\overline{0}, \overline{1} \in \mathbb{K}^{\prime}$ such that $\mathbb{K}^{\prime}$ is closed under addition and multiplication (that is, for all $a, b \in \mathbb{K}^{\prime}$ it holds that $a \oplus b \in \mathbb{K}^{\prime}$ and $\left.a \otimes b \in \mathbb{K}^{\prime}\right)$ then $\left(\mathbb{K}^{\prime}, \oplus, \otimes, \overline{0}, \overline{1}\right)$ is a subsemiring of $\mathbb{K}$.

Example 2.1. The following are examples of commutative semirings. It is easy to verify that all but the numeric semirings and the Eukasiewcz semiring are positive.

(1) The numeric semirings are $(\mathbb{Q},+, \cdot, 0,1),(\mathbb{Z},+, \cdot, 0,1)$ and $(\mathbb{N},+, \cdot, 0,1)$.

(2) The counting semiring $(\mathbb{N},+, \cdot, 0,1)$.

(3) The Boolean semiring $(\mathbb{B}, \vee, \wedge$, false, true) where $\mathbb{B}=\{$ false, true $\}$.

(4) The probability semiring ${ }^{1}\left(\mathbb{Q}^{+},+, \cdot, 0,1\right)$. Rabin [Rab63] and Segala [Seg06] define probabilistic automata over this semiring, where all transition weights must be between 0 and 1 and the sum of all transition weights starting some state, labeled by the same label must be 1 .

(5) The Viterbi semiring $([0,1], \max , \cdot, 0,1)$ is used in probabilistic parsing [DK09].

(6) The access control semiring $\mathbb{A}=(\{P<C<S<T<0\}$, min, $\max , 0, P)$, where $P$ is "public," $C$ is "confidential," $S$ is "secret," $T$ is "top secret," and 0 is "so secret that nobody can access it" [FGT08].

(7) The tropical semiring $(\mathbb{N} \cup\{\infty\}$, min $,+, \infty, 0)$ where min stands for the binary minimum function. This semiring is used in optimization problems of networks [DK09]. ${ }^{2}$

(8) The Eukasiewcz semiring, whose domain is $[0,1]$, with addition given by $x \oplus y=\max (x, y)$, with multiplication $x \otimes y=\max (0, x+y-1)$, zero element 0 , and one element 1 . This semiring is used in multivalued logics [DK09].

Complexity-wise, we use the RAM model with uniform cost measure and logarithmic word size [AH74] for our complexity results. That is, we assume that addition and multiplication of numbers, represented by a logarithmic number of bits, take constant time. Furthermore, we assume that semiring elements are encoded in binary. That is, the encoding of a semiring $\mathbb{K}$, is a function enc $: \mathbb{K} \rightarrow\{0,1\}^{*}$, which assigns a binary encoding to every semiring element. Furthermore, we denote the length ${ }^{3}$ of the encoding of an element $a \in \mathbb{K}$ by $\|a\|$. We discuss semiring encodings into more detail in Section 5.

\footnotetext{
${ }^{1}$ One may expect the domain to be $[0,1]$, but this is difficult to obtain while maintaining the semiring properties. For instance, defining $a \oplus b$ as $\min \{a+b, 1\}$ would violate distributivity.

${ }^{2}$ In literature there are actually multiple different definitions for the tropical semiring, e.g., $(\mathbb{Q} \cup$ $\{-\infty\}, \max ,+,-\infty, 0)$ and $(\mathbb{Z} \cup\{\infty\}, \min ,+, \infty, 0)$. If not mentioned otherwise, we use the tropical semiring as defined here.

${ }^{3}$ Note that we do not denote the encoding length of semiring elements by $|a|$ to obviate confusions with the absolute value function for numbers.
} 
2.2. Annotated Relations. We assume infinite and disjoint sets $\mathbf{D}$ and Vars, containing data values (or simply values) and variables, respectively. Let $V \subseteq$ Vars be a finite set of variables. A $V$-tuple is a function $\mathbf{t}: V \rightarrow \mathbf{D}$ that assigns values to variables in $V$. The arity of $\mathbf{t}$ is the cardinality $|V|$ of $V$. For a subset $X \subseteq$ Vars, we denote the restriction of $\mathbf{t}$ to the variables in $X$ by $\mathbf{t} \mid X$. We denote the set of all the $V$-tuples by $V$-Tup. We sometimes leave $V$ implicit when the precise set is not important. For the rest of this article, we assume that $(\mathbb{K}, \oplus, \otimes, \overline{0}, \overline{1})$ is a commutative semiring. A $(\mathbb{K}, \mathbf{D})$-relation $R$ over $V$ is a function $R: V$-Tup $\rightarrow \mathbb{K}$ such that its support, defined by $\operatorname{supp}(R):=\{\mathbf{t} \mid R(\mathbf{t}) \neq \overline{0}\}$, is finite. We will also write $\mathbf{t} \in R$ to abbreviate $\mathbf{t} \in \operatorname{supp}(R)$. The size of a $(\mathbb{K}, \mathbf{D})$-relation $R$ is the size of its support, that is, $|R|:=|\operatorname{supp}(R)|$. The arity of a $(\mathbb{K}, \mathbf{D})$-relation over $V$ is $|V|$. When $\mathbf{D}$ is clear from the context or irrelevant, we also use $\mathbb{K}$-relations to refer to $(\mathbb{K}, \mathbf{D})$-relations.

Example 2.2. The bottom left table in Figure 1 shows an example $(\mathbb{K}, \mathbf{D})$-relation, where $\mathbb{K}$ is the Viterbi semiring. The variables are $x_{\text {pers }}$ and $x_{\text {loc }}$, so the $V$-tuples are described in the first two columns. The third column contains the element in $\mathbb{K}$ associated to each tuple.

Green et al. [GKT07] defined a set of operators on $(\mathbb{K}, \mathbf{D})$-relations that naturally correspond to relational algebra operators and map $\mathbb{K}$-relations to $\mathbb{K}$-relations. They define the algebraic operators ${ }^{4}$ union, projection, natural join, and selection for all finite sets $V_{1}, V_{2} \subseteq$ Vars and for all $\mathbb{K}$-relations $R_{1}$ over $V_{1}$ and $R_{2}$ over $V_{2}$, as follows.

- Union: If $V_{1}=V_{2}$ then the union $R:=R_{1} \cup R_{2}$ is a function $R: V_{1}$-Tup $\rightarrow \mathbb{K}$ defined by $R(\mathbf{t}):=R_{1}(\mathbf{t}) \oplus R_{2}(\mathbf{t})$. (Otherwise, the union is not defined.)

- Projection: For $X \subseteq V_{1}$, the projection $R:=\pi_{X} R_{1}$ is a function $R: X$-Tup $\rightarrow \mathbb{K}$ defined by

$$
R(\mathbf{t}):=\bigoplus_{\mathbf{t}=\mathbf{t}^{\prime}\left\lceil X \text { and } R_{1}\left(\mathbf{t}^{\prime}\right) \neq \overline{0}\right.} R_{1}\left(\mathbf{t}^{\prime}\right) .
$$

- Natural Join: The natural join $R:=R_{1} \bowtie R_{2}$ is a function $R:\left(V_{1} \cup V_{2}\right)$-Tup $\rightarrow \mathbb{K}$ defined by

$$
R(\mathbf{t}):=R_{1}\left(\mathbf{t}_{1}\right) \otimes R_{2}\left(\mathbf{t}_{2}\right)
$$

where $\mathbf{t}_{1}$ and $\mathbf{t}_{2}$ are the restrictions $\mathbf{t} \backslash V_{1}$ and $\mathbf{t} \backslash V_{2}$, respectively.

- Selection: If $\mathbf{P}: V_{1}$-Tup $\rightarrow\{\overline{0}, \overline{1}\}$ is a selection predicate that maps each $V_{1}$-Tup $\mathbf{t}$ to either $\overline{0}$ or $\overline{1}$ then $R:=\sigma_{\mathbf{P}}\left(R_{1}\right)$ is a function $R: V_{1}$-Tup $\rightarrow \mathbb{K}$ defined by

$$
R(\mathbf{t}):=R_{1}(\mathbf{t}) \otimes \mathbf{P}(\mathbf{t}) .
$$

Proposition 2.3 [GKT07]. The above operators preserve the finiteness of the supports and therefore they map $\mathbb{K}$-relations into $\mathbb{K}$-relations.

Hence, we obtain an algebra on $\mathbb{K}$-relations.

\section{K-Annotators}

We start by setting the basic terminology. We fix a finite set $\Sigma$, which is disjoint from Vars, that we call the alphabet. A document is a finite string over the alphabet $\Sigma$, that is a finite sequence $d=\sigma_{1} \cdots \sigma_{n}$ where $\sigma_{i} \in \Sigma$ for each $i=1, \ldots, n$. By Docs we denote the set of all documents. A (k-ary) string relation is a subset of Docs $^{k}$ for some $k \in \mathbb{N}$.

\footnotetext{
${ }^{4}$ As in much of the work on semirings in provenance, e.g., Green et al. [GKT07], we do not yet consider the difference operator (which would require additive inverses).
} 


\begin{tabular}{|c|c|c|c|c|c|}
\hline$x_{\text {pers }}$ & $x_{\text {loc }}$ & annotation & $x_{\text {pers }}$ & $x_{\text {loc }}$ & annotation \\
\hline Carter & Plains, $\sqcup$ Georgia & 0.9 & {$[1,7\rangle$} & {$[13,28\rangle$} & 0.9 \\
\hline Washington & Westmoreland, $\sqcup$ Virginia & 0.9 & {$[30,40\rangle$} & {$[46,68\rangle$} & 0.9 \\
\hline Carter & Georgia, $\sqcup$ Washington & 0.81 & {$[1,7\rangle$} & {$[21,40\rangle$} & 0.81 \\
\hline Carter & Westmoreland, $\sqcup$ Virginia & 0.59049 & {$[1,7\rangle$} & {$[46,68\rangle$} & 0.59049 \\
\hline
\end{tabular}

Figure 1: A document (top), a ( $\mathbb{K}, \mathbf{D})$-relation (bottom left), and an extracted annotated span relation (bottom right).

A span identifies a substring of a document $d$ by specifying its bounding indices, that is, a span of $d$ is an expression of the form $[i, j\rangle$ where $1 \leq i \leq j \leq n+1$. By $d_{[i, j\rangle}$ we denote the substring $\sigma_{i} \cdots \sigma_{j-1}$. If $i=j$, it holds that $d_{[i, j\rangle}$ is the empty string, which we denote by $\varepsilon$. We denote by Spans $(d)$ the set of all possible spans of a document $d$ and by Spans the set of all possible spans of all possible documents. Since we will be working with relations over spans, we assume that $\mathbf{D}$ is such that Spans $\subseteq \mathbf{D}$. A $(\mathbb{K}, d)$-relation over $V \subseteq$ Vars is defined analogously to a $(\mathbb{K}, \mathbf{D})$-relation over $V$ but only uses $V$-tuples with values from $\operatorname{Spans}(d)$.

Definition 3.1. A $\mathbb{K}$-annotator (or annotator for short), is a function $S$ that is associated with a finite set $V \subseteq$ Vars of variables and maps documents $d$ into $(\mathbb{K}, d)$-relations over $V$. We denote $V$ by $\operatorname{Vars}(S)$. We sometimes also refer to an annotator as an annotator over $\mathbb{K}$ when we want to emphasize the semiring.

Notice that $\mathbb{B}$-annotators, i.e., annotators over the Boolean semiring $(\mathbb{B}, \vee, \wedge$, false, true) are simply the document spanners as defined by Fagin et al. [FKRV15].

Example 3.2. We provide an example document $d$ in Figure 1 (top). The table at the bottom right depicts a possible $(\mathbb{K}, d)$-relation obtained by a spanner that extracts (person, hometown) pairs from $d$. Notice that for each span $[i, j\rangle$ occurring in this table, the string $d_{[i, j\rangle}$ can be found in the table to the left.

In this naïve example, which is just to illustrate the definitions, we used the Viterbi semiring and annotated each tuple with $(0.9)^{k}$, where $k$ is the number of words between the spans associated to $x_{\text {pers }}$ and $x_{\text {loc }}$. The annotations can therefore be interpreted as confidence scores.

We now lift the relational algebra operators on $\mathbb{K}$-relations to the level of $\mathbb{K}$-annotators. For all documents $d$ and for all annotators $S_{1}$ and $S_{2}$ associated with $V_{1}$ and $V_{2}$, respectively, we define the following:

- Union: If $V_{1}=V_{2}$ then the union $S:=S_{1} \cup S_{2}$ is defined by $S(d):=S_{1}(d) \cup S_{2}(d) .{ }^{5}$

- Projection: For $X \subseteq V_{1}$, the projection $S:=\pi_{X} S_{1}$ is defined by $S(d):=\pi_{X} S_{1}(d)$.

- Natural Join: The natural join $S:=S_{1} \bowtie S_{2}$ is defined by $S(d):=S_{1}(d) \bowtie S_{2}(d)$.

- String selection: Let $R$ be a $k$-ary string relation. ${ }^{6}$ The string-selection operator $\sigma^{R}$ is parameterized by $k$ variables $x_{1}, \ldots, x_{k}$ in $V_{1}$ and can be written as $\sigma_{x_{1}, \ldots, x_{k}}^{R}$. Then the

\footnotetext{
${ }^{5}$ Here, $\cup$ stands for the union of two $K$-relations as was defined previously. The same is valid also for the other operators.

${ }^{6}$ Recall that a (k-ary) string relation is a subset of Docs ${ }^{k}$.
} 
annotator $S:=\sigma_{x_{1}, \ldots, x_{k}}^{R} S_{1}$ is defined as $S(d):=\sigma_{\mathbf{P}}\left(S_{1}(d)\right)$ where $\mathbf{P}$ is a selection predicate with $\mathbf{P}(\mathbf{t})=\overline{1}$ if $\left(d_{\mathbf{t}\left(x_{1}\right)}, \ldots, d_{\mathbf{t}\left(x_{k}\right)}\right) \in R$; and $\mathbf{P}(\mathbf{t})=\overline{0}$ otherwise.

Due to Proposition 2.3, it follows that the above operators form an algebra on $\mathbb{K}$-annotators.

\section{Weighted Variable-Set Automata}

In this section, we define the concept of a weighted VSet-automaton as a formalism to represent $\mathbb{K}$-annotators. This formalism is the natural generalization of VSet-automata [FKRV15] and weighted automata [DKV09].

Let $V \subseteq$ Vars be a finite set of variables. Furthermore, let $\Gamma_{V}=\{v \vdash, \dashv v \mid v \in V\}$ be the set of variable operations. ${ }^{7}$ A weighted variable-set automaton over semiring $\mathbb{K}$ (alternatively, a weighted VSet-automaton or a $\mathbb{K}$-weighted VSet-automaton) is a tuple $A:=(\Sigma, V, Q, I, F, \delta)$ where $\Sigma$ is a finite alphabet; $V \subseteq$ Vars is a finite set of variables; $Q$ is a finite set of states; $I: Q \rightarrow \mathbb{K}$ is the initial weight function; $F: Q \rightarrow \mathbb{K}$ is the final weight function; and $\delta: Q \times\left(\Sigma \cup\{\varepsilon\} \cup \Gamma_{V}\right) \times Q \rightarrow \mathbb{K}$ is a ( $\mathbb{K}$-weighted) transition function.

We define the transitions of $A$ as the set of triples $(p, o, q)$ with $\delta(p, o, q) \neq \overline{0}$. Likewise, the initial (resp., accepting) states are those states $q$ with $I(q) \neq \overline{0}$ (resp., $F(q) \neq \overline{0}$ ). A run $\rho$ of $A$ on a document $d:=d_{1} \cdots d_{n}$ is a sequence

$$
\left(q_{0}, i_{0}\right) \stackrel{o_{1}}{\rightarrow} \cdots \stackrel{o_{m}-2}{\rightarrow}\left(q_{m-1}, i_{m-1}\right) \stackrel{o_{m}-1}{\rightarrow}\left(q_{m}, i_{m}\right)
$$

where

- $I\left(q_{0}\right) \neq \overline{0}$ and $F\left(q_{m}\right) \neq \overline{0}$

- $i_{0}=1, i_{m}=n+1$, and $i_{j} \in\{1, \ldots, n\}$ for each $j \in\{1, \ldots, m-1\}$;

- each $o_{j}$ is in $\Sigma \cup\{\varepsilon\} \cup \Gamma_{V}$;

- $i_{j+1}=i_{j}$ whenever $o_{j} \in\{\varepsilon\} \cup \Gamma_{V}$ and $i_{j+1}=i_{j}+1$, otherwise;

- $\delta\left(q_{j}, o_{j}, q_{j+1}\right) \neq \overline{0}$ for all $j \geq 0$.

The weight of a run is obtained by $\otimes$-multiplying the weights of its constituent transitions. Formally, the weight $\mathrm{w}_{\rho}$ of $\rho$ is an element in $\mathbb{K}$ given by the expression

$$
I\left(q_{0}\right) \otimes \delta\left(q_{0}, o_{1}, q_{1}\right) \otimes \cdots \otimes \delta\left(q_{m-1}, o_{m-1}, q_{m}\right) \otimes F\left(q_{m}\right) .
$$

We call $\rho$ nonzero if $\mathrm{w}_{\rho} \neq \overline{0}$. A run is called valid if, for every variable $v \in V$ the following hold: there is exactly one index $i$ for which $o_{i}=v \vdash$ and exactly one index $j>i$ for which $o_{j}=\dashv v$.

For a nonzero and valid run $\rho$, we define $\mathbf{t}_{\rho}$ as the $V$-tuple that maps each variable $v \in V$ to the span $\left[i_{j}, i_{j^{\prime}}\right\rangle$ where $o_{i_{j}}=v \vdash$ and $o_{i_{j^{\prime}}}=\dashv v$. We denote the set of all valid and nonzero runs of $A$ on $d$ by

$$
\operatorname{Runs}(A, d) \text {. }
$$

We say that a weighted VSet-automaton $A$ is functional if all runs of $A$ are all valid. Note that this definition is the same as the notion of functionality of VSet-automata [FKRV15]. However, in contrast to VSet-automata, a $\mathbb{K}$-annotator over an arbitrary commutative semiring $\mathbb{K}$ might output the empty relation, even though it has multiple runs. This can be the case, as the weight of a tuple $\mathbf{t}$ is the sum of the weights of all its runs $\rho$ with $\mathbf{t}_{\rho}=\mathbf{t}$ and therefore, the weights can cancel each other out. This can not happen if $\mathbb{K}$ is positive (e.g., if $\mathbb{K}$ is the Boolean semiring).

\footnotetext{
${ }^{7}$ The operation $v \vdash$ represents opening variable $v$ and $\dashv v$ represents closing variable $v$.
} 
Notice that there may be infinitely many nonzero and valid runs of a weighted VSetautomaton on a given document, due to $\varepsilon$-cycles, which are states $q_{1}, \ldots, q_{k}$ such that $\left(q_{i}, \varepsilon, q_{i+1}\right)$ is a transition, also referred to as an $\varepsilon$-transition, for every $i \in\{1, \ldots, k-1\}$ and $q_{1}=q_{k}$. Similar to much of the standard literature on weighted automata (see, e.g., [ÉK09]) we assume that weighted VSet-automata do not have $\varepsilon$-cycles, unless mentioned otherwise. The reason for this restriction is that automata with such cycles need $\mathbb{K}$ to be closed under infinite sums for their semantics to be well-defined. ${ }^{8}$

As such, if $A$ does not have $\varepsilon$-cycles, then the result of applying $A$ on a document $d$, denoted $\llbracket A \rrbracket^{\mathbb{K}}(d)$, is the $(\mathbb{K}, d)$-relation $R$ for which

$$
R(\mathbf{t}):=\bigoplus_{\rho \in \operatorname{Runs}(A, d) \text { and } \mathbf{t}=\mathbf{t}_{\rho}} \mathrm{w}_{\rho} .
$$

Note that $\operatorname{Runs}(A, d)$ only contains runs $\rho$ that are valid and nonzero. If $\mathbf{t}$ is a $V^{\prime}$-tuple with $V^{\prime} \neq V$ then $R(\mathbf{t})=\overline{0}$, because we only consider valid runs. In addition, $\llbracket A \rrbracket^{\mathbb{K}}$ is well defined since every $V$-tuple in the support of $\llbracket A \rrbracket^{\mathbb{K}}(d)$ is a $V$-tuple over Spans $(d)$.

The size of a weighted VSet-automaton $A$ is defined by

$$
|A|:=|Q|+\sum_{q \in Q}\|I(q)\|+\sum_{q \in Q}\|F(q)\|+\sum_{p, q \in Q, a \in\left(\Sigma \cup\{\varepsilon\} \cup \Gamma_{V}\right)}\|\delta(p, a, q)\| .
$$

We say that a $\mathbb{K}$-annotator $S$ is regular if there exists a weighted VSet-automaton $A$ such that $S=\llbracket A \rrbracket^{\mathbb{K}}$. Note that this is an equality between functions. Furthermore, we say that two weighted VSet-automata $A$ and $A^{\prime}$ are equivalent if they define the same $\mathbb{K}$-annotator, that is $\llbracket A \rrbracket^{\mathbb{K}}=\llbracket A^{\prime} \rrbracket^{\mathbb{K}}$, which is the case if $\llbracket A \rrbracket^{\mathbb{K}}(d)=\llbracket A^{\prime} \rrbracket^{\mathbb{K}}(d)$ for every $d \in$ Docs. Similar to our terminology on $\mathbb{B}$-annotators, we use the term $\mathbb{B}$-weighted VSet-automata to refer to the "classical" VSet-automata of Fagin et al. [FKRV15], which are indeed weighted VSet-automata over the Boolean semiring.

We say that a $\mathbb{K}$-weighted VSet-automaton $A$ is unambiguous if, for every document $d$ and every tuple $\mathbf{t} \in \llbracket A \rrbracket^{\mathbb{K}}(d)$, there exists exactly one valid run $\rho$ of $A$ on $d$, such that $\mathbf{t}=\mathbf{t}_{\rho}$, and there is no valid run for tuples $\mathbf{t} \notin \llbracket A \rrbracket^{\mathbb{K}}(d)$. Note that, over some semirings, the class of unambiguous weighted VSet-automata is strictly contained in the class of weighted VSetautomata, as shown in the following proposition. However, over the Boolean semiring, every $\mathbb{B}$-weighted automaton can be determinized (c.f. Doleschal et al. $\left[\mathrm{DKM}^{+} 19\right.$, Proposition $4.4]^{9}$ ). Therefore there is also an unambiguous $\mathbb{B}$-weighted automaton $A_{u}$ which is equivalent to $A$, as every deterministic $\mathbb{B}$-weighted automaton is also unambiguous.

Proposition 4.1. Let $\mathbb{K}=(\mathbb{Z} \cup\{\infty\}$, min, $+, \infty, 0)$ be the tropical semiring. There is a $\mathbb{K}$ weighted VSet-automaton A such that there is no $\mathbb{K}$-weighted unambiguous VSet-automaton $A^{\prime}$ which is equivalent to $A$.

Proof. Weighted automata can be seen as weighted VSet-automata over the empty set of variables. Thus, the statement follows directly from Kirsten [Kir08, Proposition 3.2] who

\footnotetext{
${ }^{8}$ The semirings need to fulfill additional properties. Furthermore, distributivity, commutativity and associativity must also hold for infinite sums. Such semirings are called complete [Moh09].

${ }^{9}$ We note that a notion of determinism was also introduced by Maturana et al. [MRV18], but the one in $\left[\mathrm{DKM}^{+} 19\right]$ is stronger, while allowing the same expressiveness. An important difference between the two is that the containment problem for deterministic VSet-automata is PSPACE-complete when using the definition of [MRV18], whereas it is NL-complete when using the definition of $\left[\mathrm{DKM}^{+} 19\right]$. A discussion can be found in Doleschal et al. [DKM $\left.{ }^{+} 19\right]$.
} 


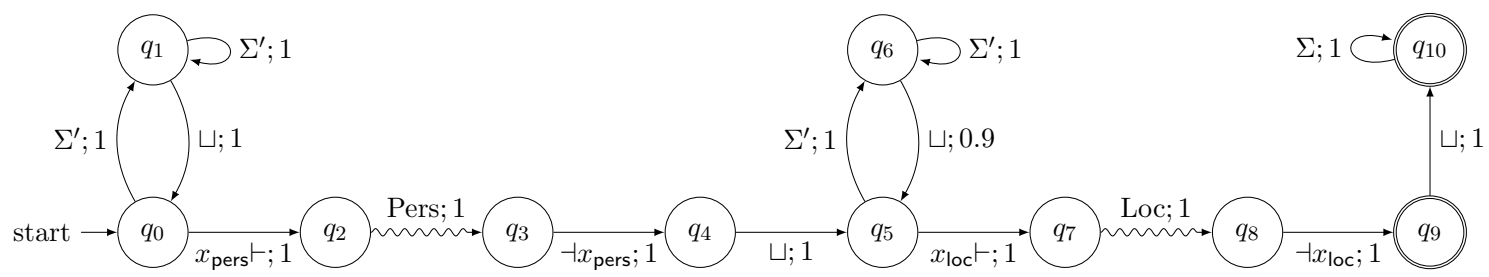

Figure 2: An example weighted VSet-automaton over the Viterbi semiring with initial state $q_{0}$ (with weight 1 ), two final states $q_{9}, q_{10}$ (both with weight 1 ), and alphabet $\Sigma^{\prime}=\Sigma \backslash\{\sqcup\}$. Pers and Loc are sub-automata matching person and location names respectively. All edges, including the edges of the sub-automata, have the weight 1 besides the transition from $q_{6}$ to $q_{5}$ with weight 0.9 .

showed that there is a $\mathbb{K}$-weighted automaton $A$ such that there is no equivalent unambiguous $\mathbb{K}$-weighted automaton $A^{\prime} .^{10}$

Example 4.2. Figure 2 shows an example weighted VSet-automaton over the Viterbi semiring, which is intended to extract (person, hometown)-tuples from a document. Here, "Pers" and "Loc" should be interpreted as sub-automata that test whether a string could be a person name or a location. (Such automata can be compiled from publicly available regular expressions ${ }^{11}$ and from deterministic rules and dictionaries as illustrated in SystemT $\left[\mathrm{CKL}^{+} 10\right]$.)

The relation extracted by this automaton from the document in Figure 1 is exactly the annotated span relation of the same figure. The weight of a tuple $\mathbf{t}$ depends on the number of spaces occurring between the span captured by $x_{\text {pers }}$ and the span captured by $x_{\text {loc }}$. More specifically the automaton assigns the weight $(0.9)^{k}$ to each tuple, where $k$ is the number of words between the two variables.

As we see next, checking equivalence of weighted VSet-automata is undecidable in general.

Proposition 4.3. Given two weighted VSet-automata $A_{1}$ and $A_{2}$ over the tropical semiring, it is undecidable to test whether $\llbracket A \rrbracket^{\mathbb{K}}=\llbracket A^{\prime} \rrbracket^{\mathbb{K}}$.

Proof. Follows directly from undecidability of the containment problem of weighted automata over the tropical semiring.(c.f. Krob [Kro94, Corollary 4.3 $]^{12}$ )

4.1. Connection to Datalog over Annotated Relations. The semantics of $\llbracket A \rrbracket^{\mathbb{K}}(d)$ is similar in spirit to the semantics of Datalog over annotated relations, studied by Deutch et al. [DMRT14], when we view the runs of $\rho \in R u n s(A, d)$ as the derivations of $\mathbf{t}$ : we take the product of the items that participate in each derivation, and sum up these products over all derivations. In fact, there is a simple translation of a weighted vset-automaton into a

\footnotetext{
${ }^{10}$ Actually, Kirsten [Kir08, Proposition 3.2] showed an even stronger result. He showed that, the result still holds if $A$ is a polynomially ambiguous weighed automaton, i.e., a weighted automaton for which the number of accepting runs of a word of length $n$ is bound by a fixed polynomial $p(n)$.

${ }^{11}$ For example, https://regexlib.com/.

${ }^{12}$ The proof by Krob is quite algebraic. See Almagor et al. [ABK11, Theorem 4] for an automata theoretic proof.
} 
Datalog program over annotated relations, similarly to the way Peterfreund et al. [PtCFK19] represent ordinary spanners via Datalog. Roughly speaking, in this translation the document $d$ is conventionally represented via the relations over the positions such as $\mathrm{O}_{\sigma}(i)$ and Successor $(i, j)$ that store the positions with the symbol $\sigma$ and successor relationship between positions, respectively (in addition to the relations First $(i)$ and Last $(i)$ that represent the first and last positions, respectively). Each tuple in these relations is annotated by $\overline{1}$. In addition, the transitions are represented by relations of the form $T_{\sigma}(q, q)$ annotated with the weight of the corresponding transition. (For simplicity, we ignore the initial and final weight functions.) The runs are derived by simple path rules such as

$$
\begin{aligned}
& \operatorname{Path}\left(x, y, q, q^{\prime}\right) \leftarrow T_{\sigma}\left(q, q^{\prime}\right), \operatorname{Successor}(x, y), \mathrm{O}_{\sigma}(y) \\
& \operatorname{Path}\left(x, y, q, q^{\prime}\right) \leftarrow \operatorname{Path}\left(x, z, q, q^{\prime \prime}\right), \operatorname{Path}\left(x, z, q^{\prime \prime}, q^{\prime}\right)
\end{aligned}
$$

where $\operatorname{Path}\left(x, y, q, q^{\prime}\right)$ states that it is possible to reach $q^{\prime}$ from $q$ when starting in $x$ and ending with $y$.

It is easy to show that a translation such as the above preserves the provenance as defined by Deutch et al. [DMRT14] (i.e., the sum of products of tuples in the derivations). We further discuss this translation, as well the general relationship between our work and that of Deutch et al. [DMRT14], in Section 9.

\section{SEMIRING ENCODINGS}

In order to state complexity results, we need to make some assumptions about the representation and computation of the semiring operations. That is, as mentioned in Section 2.1, we assume that semiring elements are encoded in binary, i.e., there is a function enc : $\mathbb{K} \rightarrow\{0,1\}^{*}$, which assigns a binary encoding to every semiring element. We write the length of the encoding of an element $a \in \mathbb{K}$ as $\|a\|$.

Throughout this article, we sometimes encode computations into matrix multiplications. To this end, we define a matrix multiplication system $M M S_{\mathbb{K}}$ of dimension $n \in \mathbb{N}$ as a triple $\operatorname{MMS}_{\mathbb{K}}:=(I, M, F)$, where $I, F \in \mathbb{K}^{n}$ are $n$-dimensional vectors over $\mathbb{K}$ and $M \in \mathbb{K}^{n \times n}$ is an $n \times n$ matrix. We define the size of a matrix multiplication system as its dimension plus the sum of the encoding lengths of all semiring elements in the system. That is,

$$
\left|\mathrm{MMS}_{\mathbb{K}}\right|=n+\sum_{a \in I}\|a\|+\sum_{a \in M}\|a\|+\sum_{a \in F}\|a\| .
$$

For an $n \times n$ matrix $X \in \mathbb{K}^{n \times n}$ (resp., a vector $X \in \mathbb{K}^{n}$ ), we define $\max (X)$ to be the maximum of the dimension of $X$ and the largest encoding length of a semiring element in $X$, that is,

$$
\max (X):=\max \left(n, \max _{x \in X}\|x\|\right) .
$$

Furthermore, for a matrix multiplication system $\mathrm{MMS}_{\mathbb{K}}=(I, M, F)$, we define

$$
\max \left(\mathrm{MMS}_{\mathbb{K}}\right)=\max (\max (I), \max (M), \max (F)) .
$$

Let $F^{T}$ be the transpose of vector $F$. By $I \times M$ we denote the matrix multiplication of $I$ and $M$. We define efficient semiring encodings as follows. 
Definition 5.1. Let $(\mathbb{K}, \oplus, \otimes, \overline{0}, \overline{1})$ be a semiring. The encoding of $\mathbb{K}$ is efficient if, for every matrix multiplication system $\mathrm{MMS}_{\mathbb{K}}$ and every natural number $k$, the encodings of the semiring elements

for all $0 \leq i \leq k$, and

$$
w_{i}:=I \times M^{i} \times F^{T},
$$

$$
w:=\bigoplus_{1 \leq i \leq k} w_{i}
$$

can be computed in time polynomial in $\left|\mathrm{MMS}_{\mathbb{K}}\right| \cdot k$.

Throughout this section, whenever we give complexity bounds, we assume that an efficient encoding of the semiring is used. As we show now, the standard encodings of most of the semirings in Example 2.1 are efficient.

Proposition 5.2. Let $(\mathbb{K}, \oplus, \otimes, \overline{0}, \overline{1})$ be a semiring. Then the encoding of $\mathbb{K}$ is efficient if, for all semiring elements $a, b \in \mathbb{K}$, the encodings of $a \oplus b$ and $a \otimes b$ can be computed in time polynomial in $\|a\|+\|b\|$ and

$$
\begin{aligned}
& \|a \oplus b\| \leq \max (\|a\|,\|b\|)+1, \text { and } \\
& \|a \otimes b\| \leq\|a\|+\|b\| .
\end{aligned}
$$

Proof. Let $\mathrm{MMS}_{\mathbb{K}}=(I, M, F)$ be a matrix multiplication system of dimension $n$ and $k \in \mathbb{N}$ be a natural number. Let $w_{1}, \ldots, w_{k}$ and $w$ be as in Definition 5.1.

We observe that the computation of $w$ requires a polynomial number of additions and multiplications. However, as the encoding of the semiring elements that are used for the computation might become large, this does not immediately imply that $w$ can be computed in time polynomial in $\left|\mathrm{MMS}_{\mathbb{K}}\right| \cdot k$.

We therefore show, for every $1 \leq i \leq k$, that the semiring elements that are required for the computation of $w_{i}$ have an encoding of size at most polynomial in $\left|\mathrm{MMS}_{\mathbb{K}}\right| \cdot k$. Due to the assumption that $\|a \oplus b\| \leq \max (\|a\|,\|b\|)+1$, this immediately implies that $\|w\|$ is polynomial in $\left|\mathrm{MMS}_{\mathbb{K}}\right| \cdot k$, which concludes the proof.

Recall that $\max (M)$ is the maximum of the dimension $n$ of $M$ and the largest representation size of any element in $M$. We begin by showing that $\max (I \times M) \leq \max (I)+\max (M)+n$ for all vectors $I \in \mathbb{K}^{n}$ and matrices $M \in \mathbb{K}^{n \times n}$. Let $x$ be an element of $I \times M$. Per definition of matrix multiplication, $x$ is the sum of $n$ elements $x_{1}, \ldots, x_{n}$, each of which is the product of an element from $I$ and an element from $M$. Thus

$$
\left\|x_{i}\right\| \leq \max (I)+\max (M)
$$

and, therefore,

$$
\|x\| \leq \max (I)+\max (M)+n .
$$

We conclude that $\max (I \times M) \leq \max (I)+\max (M)+n$ for all vectors $I \in \mathbb{K}^{n}$ and matrices $M \in \mathbb{K}^{n \times n}$.

We now show by induction that, for all $i \in \mathbb{N}$, it holds that

$$
\max \left(I \times M^{i} \times F^{T}\right) \leq \max (I)+i \cdot(\max (M)+n)+\max (F)+n,
$$

for all vectors $I, F \in \mathbb{K}^{n}$ and all matrices $M \in \mathbb{K}^{n \times n}$. Since $\max (I)+\max (M)+\max (F)+n \leq$ $\left|\mathrm{MMS}_{\mathbb{K}}\right|$, this implies that $\left\|w_{i}\right\|$ is polynomial in $\left|\mathrm{MMS}_{\mathbb{K}}\right| \cdot k$, for all $0 \leq i \leq k$.

For the base case, we observe that $w_{0}=I \times F$ is the sum of $n$ elements, each of which has size at most $\max (I)+\max (F)$. As desired, we therefore have that

$$
\max (I \times F)=\left\|w_{0}\right\| \leq \max (I)+\max (F)+n .
$$


For the inductive step, assume there is an $i \in \mathbb{N}$ such that

$$
\max \left(I \times M^{i} \times F^{T}\right) \leq \max (I)+i \cdot(\max (M)+n)+\max (F)+n
$$

for all vectors $I, F \in \mathbb{K}^{n}$ and all matrices $M \in \mathbb{K}^{n \times n}$. With $I^{\prime}:=I \times M$, we have that

$$
\max \left(I^{\prime}\right)=\max (I \times M) \leq \max (I)+\max (M)+n
$$

and, therefore,

$$
\begin{aligned}
\max \left(I \times M^{i+1} \times F^{T}\right) & =\max \left(I^{\prime} \times M^{i} \times F^{T}\right) \\
& \leq \max \left(I^{\prime}\right)+i \cdot(\max (M)+n)+\max (F)+n \\
& \leq \max (I)+\max (M)+n+i \cdot(\max (M)+n)+\max (F)+n \\
& =\max (I)+(i+1) \cdot(\max (M)+n)+\max (F)+n .
\end{aligned}
$$

This concludes the proof.

Note that all semirings over a finite domain have an efficient encoding, as each semiring element can be encoded with constant size and all operations can be carried out in constant time via a constant size lookup table.

Corollary 5.3. Each semiring $(\mathbb{K}, \oplus, \otimes, \overline{0}, \overline{1})$ where $\mathbb{K}$ is finite has an efficient encoding.

We observe that, for many semirings, the standard encodings satisfy the conditions of Proposition 5.2. Examples are the numeric semiring $(\mathbb{Z},+, \cdot, 0,1)$, the counting semiring, the Boolean semiring, the Viterbi semiring (over the rationals $\mathbb{Q}$ ), the access control semiring, and the tropical semirings. However, for some semirings, standard encodings of the semiring elements do not satisfy the conditions of Proposition 5.2. For example, consider the numeric semiring $(\mathbb{Q},+, \cdot, 0,1)$ and the encoding, where every semiring element $a=\frac{n}{d}$ is encoded by its numerator $n \in \mathbb{Z}$ and its denominator $d \in \mathbb{N}$. The problem is that the sum of two rational numbers $\frac{a}{b}, \frac{c}{d}$ is given by $x=\frac{a}{b}+\frac{c}{d}=\frac{a \cdot d+b \cdot c}{b \cdot d}$ and therefore the size of the encoding of $x$ is $\|x\| \leq\|a \cdot d\|+\|b \cdot c\|+\|c \cdot d\|$ which, in general, does not satisfy the condition that $\left\|\frac{a}{b}+\frac{c}{d}\right\| \leq \max \left(\left\|\frac{a}{b}\right\|,\left\|\frac{c}{d}\right\|\right)+1$. Even though this only increases the size of the representation by a small margin, we need some further investigation to conclude that this encoding is efficient.

Proposition 5.4. The numeric semiring $(\mathbb{Q},+, \cdot, 0,1)$ has an efficient encoding.

Proof. Let $(\mathbb{Q},+, \cdot, 0,1)$ be the numeric semiring. We assume that every semiring element $x=\frac{a}{b}$ is encoded by its numerator $a \in \mathbb{Z}$ and its denominator $b \in \mathbb{N}$. Let all numerators and denominators be encoded in binary, where two's complement encoding is used for the numerators. We observe that Proposition 5.2 holds for both encodings. Furthermore, the encoding of the denominators is monotone, that is, for every $x, y \in \mathbb{N}$ it holds that $\|x\| \leq\|y\|$ if $x \leq y$.

For a matrix multiplication system $\mathrm{MMS}_{\mathbb{K}}=(I, M, F)$, let $D$ be the set of all denominators of the rationals in $I, F$, and $M$. We will compute the least common multiple $d_{\mathrm{lcm}}$ of all denominators in $D$ and expand the representations of all numbers to the denominator $d_{\mathrm{lcm}}$. Observe that all denominators $d \in D$ are natural numbers. Therefore,

$$
\left\|d_{\mathrm{lcm}}\right\| \stackrel{(1)}{\leq}\left\|\prod_{d \in D} d\right\| \stackrel{(2)}{\leq} \sum_{d \in D}\|d\| \stackrel{(3)}{\leq}\left|\mathrm{MMS}_{\mathbb{K}}\right|,
$$


where inequality (1) follows from $d_{\mathrm{lcm}} \leq \prod_{d \in D} d$ and the monotonicity of the encoding of the denominators, inequality (2) follows from $\|x \cdot y\| \leq\|x\|+\|y\|$, and inequality (3) follows from the definition of $\left|M M S_{\mathbb{K}}\right|$. Therefore, $\left\|d_{\mathrm{lcm}}\right\| \leq\left|M M S_{\mathbb{K}}\right|$ is polynomial in $\left|\mathrm{MMS}_{\mathbb{K}}\right|$. Furthermore, the computation of $d_{\mathrm{lcm}}$ as well as the expansion can be done in polynomial time. ${ }^{13}$ We therefore assume w.l.o.g. that all rationals in $I, F$, and $M$ have the denominator $d_{\mathrm{lcm}}$.

Let $I_{\mathbb{Z}}, F_{\mathbb{Z}} \in \mathbb{Z}^{n}$ and $M_{\mathbb{Z}} \in \mathbb{Z}^{n \times n}$ be the vectors $I, F$ and the matrix $M$ where all numbers are replaced by their numerator. For all $1 \leq i \leq k$, we define

$$
w_{\mathbb{Z}, i}:=I_{\mathbb{Z}} \times M_{\mathbb{Z}}^{i} \times F_{\mathbb{Z}}^{T} .
$$

We recall that, due to Proposition $5.2, w_{\mathbb{Z}, i}$ can be computed in time polynomial in $\left|\mathrm{MMS}_{\mathbb{K}}\right| \cdot k$. Per assumption that all rationals in $I, F$, and $M$ have the denominator $d_{\mathrm{lcm}}$, we have that

$w_{i}=\frac{w_{\mathbb{Z}, i}}{d_{\mathrm{lcm}}+2}$. Furthermore, the denominator can also be computed in time polynomial in $\left|\mathrm{MMS}_{\mathbb{K}}\right|$, as $\left\|d_{\mathrm{lcm}}\right\|$ is polynomial in $\left|\mathrm{MMS}_{\mathbb{K}}\right|$ and $\|x \cdot y\| \leq\|x\|+\|y\|$ for the encoding of natural numbers. Thus, for all $i \leq k$, the encodings of the $w_{i}$ can be computed in time polynomial in $\left|\mathrm{MMS}_{\mathbb{K}}\right| \cdot k$. Furthermore, $w$ can be computed in time polynomial in $\left|\mathrm{MMS}_{\mathbb{K}}\right| \cdot k$ by first expanding all $w_{i}$ to the denominator $d_{\mathrm{lcm}}^{k+2}$ and summing up the expanded fractions. This concludes the proof.

\section{Fundamental Properties}

We now study fundamental properties of annotators. Specifically, we show that regular annotators are closed under union, projection, and join. Furthermore, annotators over a semiring $\mathbb{K}$ behave the same as document spanners with respect to string selection if $\mathbb{K}$ is positive or $\oplus$ is bipotent ${ }^{14}$ and for every $a, b \in \mathbb{K}, a \otimes b=\overline{1}$ implies that $a=b=\overline{1}$.

6.1. Epsilon Elimination. We begin the section by showing that every regular $\mathbb{K}$-annotator can be transformed into an equivalent functional regular $\mathbb{K}$-annotator without $\varepsilon$-transitions.

Proposition 6.1. For every weighted VSet-automaton $A$ there is an equivalent weighted $V$ Set-automaton $A^{\prime}$ that has no $\varepsilon$-transitions. This automaton $A^{\prime}$ can be constructed from $A$ in polynomial time. Furthermore, $A$ is functional if and only if $A^{\prime}$ is functional.

Proof. We use a result by Mohri [Moh09, Theorem 7.1] who showed that, given a weighted automaton, one can construct an equivalent weighted automaton without epsilon transitions.

More precisely, let $A=(\Sigma, V, Q, I, F, \delta)$ be a weighted VSet-automaton. Notice that $A$ can also be seen as an ordinary weighted finite state automaton $B=\left(\Sigma \cup \Gamma_{V}, Q, I, F, \delta\right)$. In this automaton, one can remove epsilon transitions by using Mohri's epsilon removal algorithm [Moh09, Theorem 7.1]. The resulting $\varepsilon$-transition free automaton $B^{\prime}=(\Sigma \cup$ $\left.\Gamma_{V}, Q^{\prime}, I^{\prime}, F^{\prime}, \delta^{\prime}\right)$ accepts the same strings as $B$. Therefore, interpreting $B^{\prime}$ as an weighted VSet-automaton $A^{\prime}=\left(\Sigma, V, Q^{\prime}, I^{\prime}, F^{\prime}, \delta^{\prime}\right)$ we have that $\llbracket A \rrbracket^{\mathbb{K}}=\llbracket A^{\prime} \rrbracket^{\mathbb{K}}$ and $A^{\prime}$ is functional if and only if $A$ is functional.

\footnotetext{
${ }^{13}$ The least common multiple can be computed using the Eucledian algorithm and the expansion of $x=\frac{a}{b}$ by multiplying the numerator $a$ by $\frac{b}{d_{1 \mathrm{~cm}}}$.

${ }^{14}$ Recall, $\oplus$ is bipotent, if $a \oplus b \in\{a, b\}$, for every $a, b \in \mathbb{K}$.
} 
Concerning complexity, Mohri shows that this algorithm runs in polynomial time, assuming that weighted- $\varepsilon$-closures can be computed in polynomial time. However, in our setting this is obvious as we do not allow $\varepsilon$-cycles. Therefore, the weight of an element of an $\varepsilon$-closure can be computed by at most $n$ matrix multiplications, where $n$ is the number of states $^{15}$ in $A$. Per assumption that $\mathbb{K}$ has an efficient encoding, these matrix multiplications can be computed in polynomial time.

6.2. Functionality. Non-functional VSet-automata are inconvenient to work with, since some of their nonzero runs are not valid and therefore do not contribute to the weight of a tuple. It is therefore desirable to be able to automatically convert weighted VSet-automata into functional weighted VSet-automata.

Proposition 6.2. Let $A$ be a weighted VSet-automaton. Then there is a functional weighted $V$ Set-automaton $A_{\text {fun }}$ that is equivalent to $A$. If $A$ has $n$ states and uses $k$ variables, then $A_{\text {fun }}$ can be constructed in time polynomial in $n$ and exponential in $k$.

Proof. The proof follows the idea of a similar result by Freydenberger [Fre19, Proposition 3.9] for unweighted VSet-automata. Like Freydenberger, we associate each state in $A_{\text {fun }}$ with a function $s: V \rightarrow\{w, o, c\}$, where $s(x)$ represents the following:

- $w$ stands for "waiting," meaning $x \vdash$ has not been read,

- o stands for "open," meaning $x \vdash$ has been read, but not $\dashv x$,

- $c$ stands for "closed," meaning $x \vdash$ and $\dashv x$ have been read.

Let $S$ be the set of all such functions. Observe that $|S|=3^{|V|}$. We now define $A_{\text {fun }}:=\left(\Sigma, V, Q_{\text {fun }}, I_{\text {fun }}, F_{\text {fun }}, \delta_{\text {fun }}\right)$ as follows:

$$
\begin{aligned}
Q_{\text {fun }} & :=Q \times S \\
I_{\text {fun }}(p, s) & := \begin{cases}I(p) & \text { where } s(x)=w \text { for all } x \in V \\
\overline{0} & \text { otherwise }\end{cases} \\
F_{\text {fun }}(p, s) & := \begin{cases}F(p) & \text { where } s(x)=c \text { for all } x \in V \\
\overline{0} & \text { otherwise }\end{cases}
\end{aligned}
$$

Furthermore, for all $(p, s) \in Q_{\text {fun }}$ and $x \in V$ we define

$$
\begin{aligned}
\delta_{\text {fun }}((p, s), a,(q, s)) & =\delta(p, a, q) & & \text { for } a \in(\Sigma \cup\{\varepsilon\}), \\
\delta_{\text {fun }}\left((p, s), x \vdash,\left(q, s_{o}^{x}\right)\right) & =\delta(p, x \vdash, q) & & \text { if } s(x)=w, \\
\delta_{\text {fun }}\left((p, s), \dashv x,\left(q, s_{c}^{x}\right)\right) & =\delta(p, \dashv x, q) & & \text { if } s(x)=o, \\
\delta_{\text {fun }}((p, s), a,(q, t)) & =\overline{0} & & \text { otherwise, }
\end{aligned}
$$

where $s_{o}^{x}$ is defined by $s_{o}^{x}(x):=o$, and $s_{o}^{x}(y):=s(y)$ for all $x \neq y$, and $s_{c}^{x}$ is defined by $s_{c}^{x}(x):=c$ and $s_{c}^{x}(y):=s(y)$ for all $x \neq y$.

Functionality follows analogously to Freydenberger [Fre19, Proposition 3.9]. It remains to show equivalence, i.e., that for every document $d \in$ Docs it holds that $\llbracket A \rrbracket^{\mathbb{K}}(d)=$ $\llbracket A_{\text {fun }} \rrbracket^{\mathbb{K}}(d)$. Observe that there is a one to one correspondence between valid nonzero runs

\footnotetext{
${ }^{15}$ As such, the construction also works in a slightly more general setting than ours, where the semiring is complete (closed under taking infinite sums, associativity, commutativity, and distributivity apply for countable sums) and weights of $\varepsilon$-closures can be computed in polynomial time.
} 
$\rho \in \operatorname{Runs}(A, d)$ and valid nonzero runs $\rho_{\text {fun }} \in \operatorname{Runs}\left(A_{\text {fun }}, d\right)$ with $\mathrm{w}_{\rho}=\mathrm{w}_{\rho_{\text {fun }}}$. Therefore, $\llbracket A \rrbracket^{\mathbb{K}}(d)=\llbracket A_{\text {fun }} \rrbracket^{\mathbb{K}}(d)$ must also hold.

The exponential blow-up in Proposition 6.2 cannot be avoided, since it already occurs for VSet-automata over the Boolean semiring. ${ }^{16}$ Functionality of VSet-automata can be checked efficiently, as we have the following result.

Proposition 6.3. Given a $\mathbb{K}$-weighted VSet-automaton $A$ with $m$ transitions and $k$ variables, it can be decided whether $A$ is functional in time $O(\mathrm{~km})$. Furthermore, $A$ is functional if and only if it is functional when interpreted as $\mathbb{B}$-weighted document spanner.

Proof. Per definition, a weighted VSet-automaton is functional if all runs are valid. Furthermore, a run $\rho$ is valid if for every variable $v \in V$ there is exactly one index $i$ for which $o_{i}=v \vdash$ and exactly one index $j>i$ for which $o_{j}=\dashv v$.

Observe that this definition only depends on the labels of the run and not on the semiring of the automaton. Therefore, a $\mathbb{K}$-weighted VSet-automaton $A$ is functional if and only if $A$ is functional when interpreted as an $\mathbb{B}$-weighted VSet-automaton $A^{\mathbb{B}}$. More formally, let $A^{\mathbb{B}}$ be the $\mathbb{B}$-weighted VSet-automaton obtained by replacing nonzero weights with true, sum by $\vee$ and multiplication by $\wedge$. The result now follows directly from Freydenberger [Fre19, Lemma 3.5], who showed that it can be verified in $O(\mathrm{~km})$ whether a VSet-automaton is functional.

6.3. Closure Under Join, Union, and Projection. We will obtain the following result.

Theorem 6.4. Regular annotators are closed under finite union, projection, and finite natural join. Furthermore, if the annotators are given as functional weighted VSet-automata, the construction for a single union, projection, and join can be done in polynomial time. Furthermore, the constructions preserve functionality.

The theorem follows immediately from Lemmata 6.5, 6.6, and 6.9. Whereas the constructions for union and projection are fairly standard, the case of join needs some care in the case that the two automata $A_{1}$ and $A_{2}$ process variable operations in different orders. ${ }^{17}$

Lemma 6.5. Given two $\mathbb{K}$-weighted VSet-automata $A_{1}$ and $A_{2}$ with $V_{1}=V_{2}$, one can construct a weighted VSet-automaton $A$ in linear time, such that $\llbracket A \rrbracket^{\mathbb{K}}=\llbracket A_{1} \rrbracket^{\mathbb{K}} \cup \llbracket A_{2} \rrbracket^{\mathbb{K}}$.

Proof. This lemma follows by the standard construction for the union of two weighted automata.

Let $A_{1}:=\left(\Sigma, V, Q_{1}, I_{1}, F_{1}, \delta_{1}\right)$ and $A_{2}:=\left(\Sigma, V, Q_{2}, I_{2}, F_{2}, \delta_{2}\right)$, such that $Q_{1} \cap Q_{2}=\emptyset$. We construct an automaton $A:=(\Sigma, V, Q, I, F, \delta)$, such that $\llbracket A \rrbracket^{\mathbb{K}}=\llbracket A_{1} \rrbracket^{\mathbb{K}} \cup \llbracket A_{2} \rrbracket^{\mathbb{K}}$. To this end, let $Q=Q_{1} \cup Q_{2}$ be the set of states, $I, F: Q \rightarrow \mathbb{K}$ with $I(q)=I_{i}(q)$ and $F(q)=F_{i}(q)$. Furthermore, let $\delta(p, a, q)=\delta_{i}(p, a, q)$ if $p, q \in Q_{i}$ and $\delta(p, a, q)=\overline{0}$ if $p, q$ are not from the state set of the same automaton. We observe that this construction can be carried out in linear time. It remains to show the correctness of the construction. To this end, observe

\footnotetext{
${ }^{16}$ Freydenberger [Fre19, Proposition 3.9] showed that there is a class of VSet-automata $\left\{A_{k} \mid k \in \mathbb{N}\right\}$ (over $\mathbb{B})$, each with one state and $k$ variables, such that every functional VSet-automaton equivalent to $A_{k}$ has at least $3^{k}$ states.

${ }^{17}$ More formally, if $A_{1}$ processes $x \vdash y \vdash a \dashv y \dashv x$ and $A_{2}$ processes $y \vdash x \vdash a \dashv x \dashv y$, then these two different sequences produce different encodings of the same tuple. This has to be considered by the automata construction.
} 
that every nonzero run $\rho$ of $A$ can only consist of states $q \in Q_{1}$ or $q \in Q_{2}$. Let $d \in$ Docs be an arbitrary document. The set Runs $(A, d)$ of all valid and nonzero runs of $A$ on $d$ is the union of two sets $P_{1}(A, d), P_{2}(A, d)$, where a run $\rho$ is in $P_{i}(A, d)$ if it consists of states in $Q_{i}$. Furthermore, it holds that $\rho \in P_{i}(A, d)$ if and only if $\rho \in R u n s\left(A_{i}, d\right)$ and therefore,

$$
\begin{aligned}
& \llbracket A \rrbracket^{\mathbb{K}}(d)(\mathbf{t})=\underset{\rho \in \operatorname{Runs}(A, d) \text { and } \mathbf{t}=\mathbf{t}_{\rho}}{\mathrm{w}_{\rho}} \\
& =\left(\bigoplus_{\rho \in P_{1}(A, d) \text { and } \mathbf{t}=\mathbf{t}_{\rho}} \mathrm{w}_{\rho}\right) \oplus\left(\bigoplus_{\rho \in P_{2}(A, d) \text { and } \mathbf{t}=\mathbf{t}_{\rho}} \mathrm{w}_{\rho}\right) \\
& =\left(\bigoplus_{\rho \in \operatorname{Runs}\left(A_{1}, d\right) \text { and } \mathbf{t}=\mathbf{t}_{\rho}} \mathrm{w}_{\rho}\right) \oplus\left(\bigoplus_{\rho \in \operatorname{Runs}\left(A_{2}, d\right) \text { and } \mathbf{t}=\mathbf{t}_{\rho}} \mathrm{w}_{\rho}\right) \\
& =\llbracket A_{1} \rrbracket^{\mathbb{K}}(d)(\mathbf{t}) \oplus \llbracket A_{2} \rrbracket^{\mathbb{K}}(d)(\mathbf{t}) .
\end{aligned}
$$

This concludes the proof that $\llbracket A \rrbracket^{\mathbb{K}}=\llbracket A_{1} \rrbracket^{\mathbb{K}} \cup \llbracket A_{2} \rrbracket^{\mathbb{K}}$.

Lemma 6.6. Given a $\mathbb{K}$-weighted VSet-automaton $A$ and a subset $X \subseteq V$ of the variables $V$ of $A$, there exists a weighted VSet-automaton $A^{\prime}$ with $\llbracket A^{\prime} \rrbracket^{\mathbb{K}}=\pi_{X} \llbracket A \rrbracket^{\mathbb{K}}$. Furthermore, if $A$ is functional, then $A^{\prime}$ can be constructed in polynomial time.

Proof. Let $A:=(\Sigma, V, Q, I, F, \delta)$ and $V^{-}=V \backslash X$. If $A$ is not yet functional, we can assume by Proposition 6.2 that it is, at exponential cost in the number of variables of $A$. Furthermore, assume that, for every nonzero transition, there is a run $\rho$ which uses the transition. Due to $A$ being functional, we will be able to construct $A^{\prime}$ by replacing all transitions labeled with a variable operation $o \in \Gamma_{V^{-}}$with an $\varepsilon$-transition of the same weight. More formally, let $A^{\prime}:=\left(\Sigma, X, Q, I, F, \delta^{\prime}\right)$, such that

- $\delta^{\prime}(p, o, q)=\delta(p, o, q)$ for all $p, q \in Q$ and $o \in \Sigma \cup\{\varepsilon\} \cup \Gamma_{X}$, and

- $\delta^{\prime}(p, \varepsilon, q)=\delta(p, o, q)$ for all $p, q \in Q$ and $o \in \Gamma_{V^{-}}$.

We first argue why $\delta^{\prime}$ is well defined. Towards a contradiction, assume that $\delta^{\prime}$ is not well-defined. This can only happen if $A$ has two transitions $\delta\left(p, o_{1}, q\right)$ and $\delta\left(p, o_{2}, q\right)$ with $o_{1}, o_{2} \in \Gamma_{V^{-}} \cup\{\varepsilon\}$ and $o_{1} \neq o_{2}$. Therefore, there are two runs $\rho_{1}, \rho_{2}$ of A, which only differ on this transition, that is, $\rho_{1}$ uses $\delta\left(p, o_{1}, q\right)$ and $\rho_{2}$ uses $\delta\left(p, o_{2}, q\right)$ respectively. Since $o_{1} \neq o_{2}$ and $o_{1}, o_{2} \in \Gamma_{V^{-}} \cup\{\varepsilon\}$, either $\rho_{1}$ or $\rho_{2}$ are not valid, contradicting functionality of $A$.

It remains to show that $\llbracket A^{\prime} \rrbracket^{\mathbb{K}}=\pi_{X} \llbracket A \rrbracket^{\mathbb{K}}$. To this end, let $d \in$ Docs be an arbitrary document. Every run $\rho$ of $A$ selecting $\mathbf{t}$ on $d$ corresponds to exactly one run $\rho^{\prime}$ of $A^{\prime}$ selecting $\mathbf{t}^{\prime}$ on $d$ such that $\mathbf{t}^{\prime}=\mathbf{t}\left\lceil X\right.$ and $\mathrm{w}_{\rho}=\mathrm{w}_{\rho^{\prime}}$. Therefore,

$$
\begin{aligned}
\pi_{X} \llbracket A \rrbracket^{\mathbb{K}}(d)\left(\mathbf{t}^{\prime}\right)= & \bigoplus_{\mathbf{t}^{\prime}=\mathbf{t}\left\lceil X \text { and } \llbracket A \rrbracket^{\mathbb{K}}(d)(\mathbf{t}) \neq \overline{0}\right.} \llbracket A \rrbracket^{\mathbb{K}}(d)(\mathbf{t}) \\
= & \bigoplus_{\mathbf{t}^{\prime}=\mathbf{t}\left\lceil X \text { and } \llbracket A \rrbracket^{\mathbb{K}}(d)(\mathbf{t}) \neq \overline{0}\right.} \bigoplus_{\rho \in \operatorname{Runs}(A, d) \text { and } \mathbf{t}=\mathbf{t}_{\rho}} \mathbf{w}_{\rho} \\
= & \bigoplus_{\rho^{\prime} \in \operatorname{Runs}\left(A^{\prime}, d\right) \text { and } \mathbf{t}^{\prime}=\mathbf{t}_{\rho^{\prime}}} \mathrm{w}_{\rho^{\prime}} \\
= & \llbracket A^{\prime} \rrbracket^{\mathbb{K}}(d)\left(\mathbf{t}^{\prime}\right) .
\end{aligned}
$$


Therefore, $\llbracket A^{\prime} \rrbracket^{\mathbb{K}}=\pi_{X} \llbracket A \rrbracket^{\mathbb{K}}$.

We will now show that regular annotators are closed under join. Freydenberger et al. [FKP18, Lemma 3.10], showed that, given two functional $\mathbb{B}$-weighted VSet-automata $A_{1}$ and $A_{2}$, one can construct a functional VSet-automaton $A$ with $\llbracket A \rrbracket^{\mathbb{B}}=\llbracket A_{1} \rrbracket^{\mathbb{B}} \bowtie \llbracket A_{2} \rrbracket^{\mathbb{B}}$ in polynomial time. The construction is based on the classical product construction for the intersection of NFAs. However, $A_{1}$ and $A_{2}$ can process consecutive variable operations in different orders which must be considered during the construction. To deal with this issue, we adapt and combine multiple constructions from the literature.

To be precise, we adopt so called extended VSet-automata as defined by Amarilli et al. [ABMN19] by adding weights to the transitions. ${ }^{18}$ An extended $\mathbb{K}$-weighted VSetautomaton on alphabet $\Sigma$ and variable set $V$ is an automaton $A_{E}=(\Sigma, V, Q, I, F, \delta)$, where $Q=Q_{v} \uplus Q_{\ell}$ is a disjoint union of variable states $Q_{v}$ and letter states $Q_{\ell}$. Furthermore, $I: Q \rightarrow \mathbb{K}$ is the initial weight function, such that $I(q)=\overline{0}$, for every $q \in Q_{\ell}$. Analogously, $F: Q \rightarrow \mathbb{K}$ is a final weight function, such that $F(q)=\overline{0}$, for every $q \in Q_{v}$. Finally, we define the (partial) transition function $\delta: Q \times\left(\Sigma \cup 2^{\Gamma_{V}}\right) \times Q \rightarrow \mathbb{K}$, such that transitions labeled by $\sigma \in \Sigma$ originate in letter states and terminate in variable states and $T \subseteq \Gamma_{V}$ transitions are between variable states and letter states. More formally, for every $\sigma \in \Sigma$, it holds that if $\delta(p, \sigma, q) \neq \overline{0}$ then $p \in Q_{\ell}$ and $q \in Q_{v}$. Furthermore, for every $T \subseteq \Gamma_{V}$, if $\delta(p, T, q) \neq \overline{0}$ then $p \in Q_{v}$ and $q \in Q_{\ell}$.

We define runs of extended weighted VSet-automata analogously to runs on weighted VSet-automata. That is, a run $\rho$ of $A_{E}$ on a document $d:=d_{1} \cdots d_{n}$ is a sequence

$$
\left(q_{0}, i_{0}\right) \stackrel{o_{1}}{\rightarrow} \cdots \stackrel{o_{m-1}}{\rightarrow}\left(q_{m-1}, i_{m-1}\right) \stackrel{o_{m}}{\rightarrow}\left(q_{m}, i_{m}\right)
$$

where

- $I\left(q_{0}\right) \neq \overline{0}$ and $F\left(q_{m}\right) \neq \overline{0}$

- $i_{0}=1, i_{m}=n+1$, and $i_{j} \in\{1, \ldots, n\}$ for each $j \in\{1, \ldots, m-1\}$;

- each $o_{j}$ is in $\Sigma \cup\{\varepsilon\} \cup 2^{\Gamma_{V}}$;

- $i_{j+1}=i_{j}$ whenever $o_{j} \in\{\varepsilon\} \cup 2^{\Gamma_{V}}$ and $i_{j+1}=i_{j}+1$, otherwise;

- $\delta\left(q_{j}, o_{j}, q_{j+1}\right) \neq \overline{0}$ for all $j \geq 0$.

The weight $\mathrm{w}_{\rho}$ of a run $\rho$ on an extended weighted VSet-automaton, $\llbracket A_{E} \rrbracket^{\mathbb{K}}$, functionality, and unambiguity are defined analogously to the weighted VSet-automata.

Proposition 6.7. For every functional weighted VSet-automaton A, there exists an equivalent functional extended weighted VSet-automaton $A_{E}$ and vice versa. Given an automaton in one model, one can construct an automaton in the other model in polynomial time. Furthermore, the conversion preserves unambiguity.

Proof. Let $A:=(\Sigma, V, Q, I, F, \delta)$ be a weighted functional VSet-automaton.

Due to Proposition 6.3 a weighted VSet-automaton is functional if and only if the automaton $A$ interpreted as $\mathbb{B}$-weighted VSet-automaton is functional. For functional VSet-automata it is well known ${ }^{19}$ that there is a function $s: Q \times V \rightarrow\{w, o, c\}$, where

- $s(q, v)=w$ stands for "waiting," meaning that no run $\rho$ of $A$ such that $v \vdash$ is read before reaching state $q$.

- $s(q, v)=o$ stands for "open," meaning that all runs $\rho$ of $A$ read $v \vdash$ but not $\dashv v$ before reaching state $q$.

\footnotetext{
${ }^{18}$ We note that extended VSet-automata have been originally introduced in Florenzano et al. $\left[\mathrm{FRU}^{+} 18\right]$

${ }^{19}$ For example, compare Freydenberger [Fre19], Freydenberger et al. [FKP18].
} 
- $s(q, v)=c$ stands for "closed," meaning that all runs $\rho$ of $A$ read $v \vdash$ and $\dashv v$ before reaching state $q$.

Based on $s$, we define the function $S: Q \times Q \rightarrow \Gamma_{V}$, such that $S\left(q, q^{\prime}\right)=T$, if on every run $\rho$ of $A$ which visits $q^{\prime}$ after $q$, exactly the variable operations $T$ must be read between $q$ and $q^{\prime}$. More formally, $x \vdash \in S(p, q)$ if and only if $s(p, x)=w$ and $s(q, x) \neq w$ and $\dashv x \in S(p, q)$ if and only if $s(p, x) \neq c$ and $s(q, x)=c$.

We assume, w.l.o.g., that the states of $A$ are $\{1, \ldots, n\}$ for some $n \in \mathbb{N}$. For every state $i \in Q$, we define the vector $V_{i}$, where

$$
V_{i}(j)= \begin{cases}\overline{0} & \text { if } i \neq j \\ \overline{1} & \text { if } i=j\end{cases}
$$

Furthermore, we define the $n \times n$ matrix $M_{p, q}$ where

$$
M_{p, q}(i, j)= \begin{cases}\delta(i, o, j) & \text { if } o \in S(p, q) \\ \overline{1} & \text { otherwise } .\end{cases}
$$

We construct the weighted extended functional VSet-automaton $A_{E}:=\left(\Sigma, V, Q_{\ell} \cup\right.$ $\left.Q_{v}, I_{E}, F_{E}, \delta_{E}\right)$ as follows. Let $Q_{\ell}:=\left\{q_{\ell} \mid q \in Q\right\}$ and $Q_{v}:=\left\{q_{v} \mid q \in Q\right\}$ be two disjoint copies of the states of $A$. Furthermore, let

$$
\begin{gathered}
I_{E}(q):= \begin{cases}I(q) & \text { if } q \in Q_{v} \\
\overline{0} & \text { if } q \in Q_{\ell}\end{cases} \\
F_{E}(q):= \begin{cases}\overline{0} & \text { if } q \in Q_{v} \\
F(q) & \text { if } q \in Q_{\ell}\end{cases}
\end{gathered}
$$

We define $\delta_{E}$ as follows

$$
\begin{aligned}
\delta_{E}\left(p_{l}, \sigma, q_{v}\right) & :=\delta(p, \sigma, q) & & \text { for all } \sigma \in \Sigma, \\
\delta_{E}\left(p_{v}, O, q_{\ell}\right) & :=V_{p_{v}} \times\left(M_{p_{v}, q_{\ell}}\right)^{|O|} \times V_{q_{\ell}}^{T} & & \text { for } O=S\left(p_{v}, q_{\ell}\right) \\
\delta_{E}\left(p_{v}, \emptyset, p_{\ell}\right) & :=\overline{1} & &
\end{aligned}
$$

We observe that per assumption that $\mathbb{K}$ has an efficient encoding, it follows that $A_{E}$ can be constructed in polynomial time. It remains to show that $\llbracket A \rrbracket^{\mathbb{K}}=\llbracket A_{E} \rrbracket^{\mathbb{K}}$. To this end, we define a function, which maps valid runs of $A$ to runs of $A_{E}$. More formally, let

$$
\left(q_{0}, i_{0}\right) \stackrel{o_{1}}{\rightarrow} \cdots \stackrel{o_{m-2}}{\rightarrow}\left(q_{m-1}, i_{m-1}\right) \stackrel{o_{m}-1}{\rightarrow}\left(q_{m}, i_{m}\right)
$$

be a run $\rho$ of $A$ on $d=d_{1} \cdots d_{n}$.

Let $q_{v}^{1} \in Q_{v}$ (resp., $q_{\ell}^{n+1} \in Q_{\ell}$ ) be the variable state (resp., letter state) corresponding to $q_{0}$ (resp., $q_{m}$ ). Furthermore, for $1 \leq k \leq n$, let $q_{\ell}^{k}, q_{v}^{k+1}$ be the states corresponding to the states visited by $\rho$ while reading the symbol $d_{k}$. That is, for $\left(q_{j}, k\right) \stackrel{d_{k}}{\rightarrow}\left(q_{j+1}, k+1\right)$ in $\rho$, $q_{\ell}^{k-1}$ corresponds to $q_{j}$ and $q_{v}^{k}$ to $q_{j+1}$.

We define $f(\rho)$ as the run $\rho_{E} \in \operatorname{Runs}\left(A_{E}, d\right)$ such that

$$
\rho_{E}=\left(q_{v}^{1}, 1\right) \stackrel{S\left(q_{v}^{1}, q_{\ell}^{1}\right)}{\longrightarrow}\left(q_{\ell}^{1}, 1\right) \stackrel{d_{1}}{\longrightarrow}\left(q_{v}^{2}, 2\right) \stackrel{S\left(q_{v}^{2}, q_{\ell}^{2}\right)}{\longrightarrow} \cdots \stackrel{d_{n}}{\longrightarrow}\left(q_{v}^{n+1}, n+1\right) \stackrel{S\left(q_{v}^{n+1}, q_{\ell}^{n+1}\right)}{\longrightarrow}\left(q_{\ell}^{n+1}, n+1\right) .
$$


For every valid run $\rho_{E} \in \operatorname{Runs}\left(A_{E}, d\right)$, it holds that $\mathrm{w}_{\rho_{E}}=\bigoplus_{\rho \in \operatorname{Runs}(A, d) \text { with } f(\rho)=\rho_{E}} \mathrm{w}_{\rho}$. Therefore, it follows that

$$
\begin{aligned}
\llbracket A_{E} \rrbracket^{\mathbb{K}}(d)(\mathbf{t}) & =\bigoplus_{\rho_{E} \in \operatorname{Runs}\left(A_{E}, d\right) \text { and } \mathbf{t}=\mathbf{t}_{\rho_{E}}} \mathrm{w}_{\rho_{E}} \\
& =\bigoplus_{\rho_{E} \in \operatorname{Runs}\left(A_{E}, d\right) \text { and } \mathbf{t}=\mathbf{t}_{\rho_{E}}} \rho \in \operatorname{Runs}(A, d) \text { with } f(\rho)=\rho_{E} \\
& =\bigoplus_{\rho \in \operatorname{Runs}(A, d) \text { and } \mathbf{t}=\mathbf{t}_{\rho}} \mathrm{w}_{\rho} \\
& =\llbracket A \rrbracket^{\mathbb{K}}(d)(\mathbf{t}) .
\end{aligned}
$$

It remains to show that $A_{E}$ is unambiguous if $A$ is unambiguous. To this end, assume that $A_{E}$ is not unambiguous. Thus, there must be two runs $\rho_{E}^{1} \neq \rho_{E}^{2}$ on $A_{E}$, encoding the same tuple. By construction of $A_{E}$, there must be two runs $\rho_{1} \neq \rho_{2}$ of $A$ which encode the same tuple, however this contradicts the unambiguity of $A$. Therefore $A_{E}$ must be unambiguous.

For the other direction, one can construct a weighted VSet-automaton $A$ with $\varepsilon$ transitions, ${ }^{20}$ by replacing every edge $\delta(p, T, q)=w$ by a sequence of transitions $\delta\left(p, v_{1}, q_{1}\right)=$ $w, \delta\left(q_{1}, v_{2}, q_{2}\right)=\overline{1}, \ldots, \delta\left(q_{n-1}, v_{n}, q\right)=\overline{1}$, where $T=\left\{v_{1}, \ldots, v_{n}\right\}$ and $q_{1}, \ldots, q_{n-1}$ are new states. We observe that only the first transition has weight $w$, whereas all other transitions have weight $\overline{1}$. This construction also runs in polynomial time and it is straightforward to verify that $\llbracket A \rrbracket^{\mathbb{K}}=\llbracket A_{E} \rrbracket^{\mathbb{K}}$ and that $A$ is unambiguous if $A_{E}$ is unambiguous.

Proposition 6.8. Let $A_{1}, A_{2}$ be two functional extended $\mathbb{K}$-weighted VSet-automata. One can construct a functional extended $\mathbb{K}$-weighted VSet-automaton $A$ in polynomial time, such that $\llbracket A \rrbracket^{\mathbb{K}}=\llbracket A_{1} \rrbracket^{\mathbb{K}} \bowtie \llbracket A_{2} \rrbracket^{\mathbb{K}}$. Furthermore, $A$ is unambiguous if $A_{1}$ and $A_{2}$ are unambiguous.

Proof. Let $A_{1}=\left(\Sigma, V_{1}, Q_{1}, I_{1}, F_{1}, \delta_{1}\right)$ and $A_{2}=\left(\Sigma, V_{2}, Q_{2}, I_{2}, F_{2}, \delta_{2}\right)$ be two $\mathbb{K}$-weighted extended functional VSet-automata. We construct a $\mathbb{K}$-weighted extended functional VSetautomaton $A=\left(\Sigma, V_{1} \cup V_{2}, Q_{1} \times Q_{2}, I, F, \delta\right)$ such that $\llbracket A \rrbracket^{\mathbb{K}}=\llbracket A_{1} \rrbracket^{\mathbb{K}} \bowtie \llbracket A_{2} \rrbracket^{\mathbb{K}}$. To this end, let $I\left(q_{1}, q_{2}\right)=I_{1}\left(q_{1}\right) \otimes I_{2}\left(q_{2}\right)$ and $F\left(q_{1}, q_{2}\right)=F_{1}\left(q_{1}\right) \otimes F_{2}\left(q_{2}\right)$. Furthermore, let

$$
\delta\left(\left(p_{1}, p_{2}\right), \sigma,\left(q_{1}, q_{2}\right)\right)=\delta_{1}\left(p_{1}, \sigma, q_{1}\right) \otimes \delta_{2}\left(p_{2}, \sigma, q_{2}\right),
$$

if $\sigma \in \Sigma$, and otherwise, if $T \subseteq \Gamma_{V}$,

$$
\delta\left(\left(p_{1}, p_{2}\right), T,\left(q_{1}, q_{2}\right)\right)=\delta_{1}\left(p_{1}, T \cap \Gamma_{V_{1}}, q_{1}\right) \otimes \delta_{2}\left(p_{2}, T \cap \Gamma_{V_{2}}, q_{2}\right) .
$$

We observe that $A$ can be constructed in polynomial time. We have to show that $\llbracket A \rrbracket^{\mathbb{K}}=$ $\llbracket A_{1} \rrbracket^{\mathbb{K}} \bowtie \llbracket A_{2} \rrbracket^{\mathbb{K}}$. Let $d \in$ Docs be a document and $\mathbf{t}$ be a tuple. Every run $\rho \in \operatorname{Runs}(A, d)$ with $\mathbf{t}_{\rho}=\mathbf{t}$ originates from of a set of runs $\rho_{1} \in \operatorname{Runs}\left(A_{1}, d\right)$ selecting $\pi_{V_{1}} \mathbf{t}$ and a set of runs $\rho_{2} \in \operatorname{Runs}\left(A_{2}, d\right)$ selecting $\pi_{V_{2}} \mathbf{t}$. Due to distributivity of $\otimes$ over $\oplus$, it holds that $\mathrm{w}_{\rho}=\mathrm{w}_{\rho_{1}} \otimes \mathrm{w}_{\rho_{2}}$. Furthermore, every run in $A$ corresponds to exactly one run in $A_{1}$ and one run in $A_{2}$. It follows directly that $\llbracket A \rrbracket^{\mathbb{K}}(d)(\mathbf{t})=\llbracket A_{1} \rrbracket^{\mathbb{K}}(d)\left(\pi_{V_{1}} \mathbf{t}\right) \otimes \llbracket A_{2} \rrbracket^{\mathbb{K}}(d)\left(\pi_{V_{2}} \mathbf{t}\right)$ and that the construction preserves unambiguity.

We now show that regular annotators are closed under join.

\footnotetext{
${ }^{20}$ By Proposition 6.1 the $\varepsilon$-transitions can be removed in polynomial time.
} 
Lemma 6.9. Given two $\mathbb{K}$-weighted VSet-automata $A^{1}$ and $A^{2}$, one can construct a weighted functional VSet-automaton $A$ with $\llbracket A \rrbracket^{\mathbb{K}}=\llbracket A^{1} \rrbracket^{\mathbb{K}} \bowtie \llbracket A^{2} \rrbracket^{\mathbb{K}}$. Furthermore, $A$ can be constructed in polynomial time if $A^{1}$ and $A^{2}$ are functional and $A$ is unambiguous if $A_{1}$ and $A_{2}$ are unambiguous.

Proof. If $A^{1}$ and $A^{2}$ are not yet functional, we can assume that they are at an exponential cost in their number of variables (cf. Proposition 6.2). By Proposition 6.7, one can construct functional extended weighted VSet-automata $A_{E}^{1}, A_{E}^{2}$ with $\llbracket A^{i} \rrbracket^{\mathbb{K}}=\llbracket A_{E}^{i} \rrbracket^{\mathbb{K}}$. Furthermore, due to Proposition 6.8, one can construct a functional extended weighted VSet-automaton $A_{E}$ with $\llbracket A_{E} \rrbracket^{\mathbb{K}}=\llbracket A_{E}^{1} \rrbracket^{\mathbb{K}} \bowtie \llbracket A_{E}^{2} \rrbracket^{\mathbb{K}}$. Thus, again applying Proposition 6.7 , one can construct a functional weighted VSet-automaton $A_{E}$ with $\llbracket A_{E} \rrbracket^{\mathbb{K}}=\llbracket A_{E}^{1} \rrbracket^{\mathbb{K}} \bowtie \llbracket A_{E}^{2} \rrbracket^{\mathbb{K}}=\llbracket A^{1} \rrbracket^{\mathbb{K}} \bowtie \llbracket A^{2} \rrbracket^{\mathbb{K}}$. Note that all constructions are in polynomial time if $A^{1}$ and $A^{2}$ are functional and preserve unambiguity. Thus, concluding the proof with $A:=A_{E}$.

The previous lemma also has applications to unambiguous functional VSet-automata over the Boolean semiring.

Corollary 6.10. Given two unambiguous functional VSet-automata $A_{1}, A_{2}$ over the Boolean semiring, one can construct an unambiguous functional VSet-automaton $A$ with $\llbracket A \rrbracket^{\mathbb{B}}=$ $\llbracket A_{1} \rrbracket^{\mathbb{B}} \bowtie \llbracket A_{2} \rrbracket^{\mathbb{B}}$ in polynomial time.

6.4. Closure Under String Selection. A $k$-ary string relation is recognizable if it is a finite union of Cartesian products $L_{1} \times \cdots \times L_{k}$, where each $L_{i}$ is a regular language over $\Sigma$ [Sak09]. Let $\mathrm{REG}_{\mathbb{K}}$ be the set of regular $\mathbb{K}$-annotators. We say that a $k$-ary string relation ${ }^{21}$ $R$ is selectable by regular $\mathbb{K}$-annotators if the class of $\mathbb{K}$-annotators is closed under the string selection $\sigma^{R}$. More formally:

$$
\left\{\sigma_{x_{1}, \ldots, x_{k}}^{R}(S) \mid S \in \mathrm{REG}_{\mathbb{K}} \text { and } x_{i} \in \operatorname{Vars}(S) \text { for all } 1 \leq i \leq k\right\} \subseteq \mathrm{REG}_{\mathbb{K}},
$$

that is, the class of $\mathbb{K}$-annotators is closed under selection using $R$. If $\mathbb{K}=\mathbb{B}$, we say that $R$ is selectable by document spanners. Fagin et al. [FKRV15] proved that a string relation is recognizable if and only if it is selectable by document spanners. Here, we generalize this result in the context of weights and annotation. Indeed, it turns out that the equivalence is maintained for all positive semirings.

Theorem 6.11. Let $(\mathbb{K}, \oplus, \otimes, \overline{0}, \overline{1})$ be a positive semiring and $R$ be a string relation. The following are equivalent:

(1) $R$ is recognizable.

(2) $R$ is selectable by document spanners.

(3) $R$ is selectable by $\mathbb{K}$-annotators.

Proof. The equivalence between (1) and (2) is known [FKRV15, Theorem 4.16]. We will show the implication $(2) \Rightarrow(3)$ in Lemma 6.18 and implication (3) to (2) in Lemma 6.19.

As we will see, the proof of Lemma 6.18 is heavily based on the closure properties from Theorem 6.4 and holds beyond positive semirings. For the proof of Lemma 6.19, we use semiring morphisms to turn $\mathbb{K}$-weighted VSet-automata into $\mathbb{B}$-weighted VSet-automata and need positivity of the semiring. We need some preliminary results in order to give the proofs of the lemma.

\footnotetext{
${ }^{21}$ Recall that a $k$-ary string relation is a subset of Docs ${ }^{k}$.
} 
Definition 6.12. Let $R$ be a $k$-ary string relation. A $\mathbb{K}$-weighted VSet-automaton $A_{R}^{\mathbb{K}}$ with variables $\left\{x_{1}, \ldots, x_{k}\right\}$ selects $R$ over $\mathbb{K}$ if for every document $d \in$ Docs and every tuple $\mathbf{t}$ it holds that $\llbracket A_{R}^{\mathbb{K}} \rrbracket^{\mathbb{K}}(d)(\mathbf{t})=\overline{1}$ if $\left(d_{\mathbf{t}\left(x_{1}\right)}, \ldots, d_{\mathbf{t}\left(x_{k}\right)}\right) \in R$, and $\overline{0}$, otherwise, if $\left(d_{\mathbf{t}\left(x_{1}\right)}, \ldots, d_{\mathbf{t}\left(x_{k}\right)}\right) \notin R$.

Lemma 6.13. Let $R$ be a $k$-ary string relation. Then $R$ is selectable by $\mathbb{K}$-annotators if and only if there is a VSet-automaton $A_{R}^{\mathbb{K}}$ that selects $R$ over $\mathbb{K}$.

Proof. Assume that $R$ is selectable by $\mathbb{K}$-annotators. Let $A$ be the $\mathbb{K}$-weighted VSetautomaton that assigns weight $\overline{1}$ to all possible tuples for all documents. As $R$ is selectable by $\mathbb{K}$-annotators, $\sigma_{x_{1}, \ldots, x_{k}}^{R}\left(\llbracket A \rrbracket^{\mathbb{K}}\right)$ must be a regular $\mathbb{K}$-annotator. Thus, the $\mathbb{K}$-weighted VSet-automaton $A_{R}^{\mathbb{K}}$ representing $\sigma_{x_{1}, \ldots, x_{k}}^{R}\left(\llbracket A \rrbracket^{\mathbb{K}}\right)$ selects $R$ over $\mathbb{K}$.

For the other direction, let $A_{R}^{\mathbb{K}}$ be as defined. Let $A$ be a $\mathbb{K}$-weighted VSet-automaton. Per definition of string selection, $\sigma_{x_{1}, \ldots, x_{k}}^{R}\left(\llbracket A \rrbracket^{\mathbb{K}}\right)(\mathbf{t})=\llbracket A \rrbracket^{\mathbb{K}}(\mathbf{t}) \otimes \overline{0}=\overline{0}$ if $\left(d_{\mathbf{t}\left(x_{1}\right)}, \ldots, d_{\mathbf{t}\left(x_{k}\right)}\right) \notin$ $R$ and $\sigma_{x_{1}, \ldots, x_{k}}^{R}\left(\llbracket A \rrbracket^{\mathbb{K}}\right)(\mathbf{t})=\llbracket A \rrbracket^{\mathbb{K}}(\mathbf{t}) \otimes \overline{1}=\llbracket A \rrbracket^{\mathbb{K}}(\mathbf{t})$, otherwise. Therefore, $\sigma_{x_{1}, \ldots, x_{k}}^{R}\left(\llbracket A \rrbracket^{\mathbb{K}}\right)=$ $\llbracket A \rrbracket^{\mathbb{K}} \bowtie \llbracket A_{R}^{\mathbb{K}} \rrbracket^{\mathbb{K}}$, which proves that $R$ is selectable by $\mathbb{K}$-annotators, as $\mathbb{K}$-annotators are closed under join (c.f. Theorem 6.4).

We will now define means of transferring the structure of weighted automata between different semirings, that is, we define $\mathbb{B}$-projections and $\mathbb{K}$-extensions of weighted VSetautomata.

Definition 6.14. Let $A$ be a weighted VSet-automaton over $\mathbb{K}$. A $\mathbb{B}$-weighted VSetautomaton $A^{\mathbb{B}}$ is a $\mathbb{B}$-projection of $A$ if, for every document $d \in$ Docs, it holds that $\mathbf{t} \in \llbracket A^{\mathbb{B}} \rrbracket^{\mathbb{B}}(d) \Leftrightarrow \mathbf{t} \in \llbracket A \rrbracket^{\mathbb{K}}(d)$.

Definition 6.15. Let $A$ be a $\mathbb{B}$-weighted VSet-automaton. Then a $\mathbb{K}$-weighted VSetautomaton $A^{\mathbb{K}}$ is called a $\mathbb{K}$-extension of $A$ if, for every document $d \in$ Docs and every tuple $\mathbf{t}$, the following are equivalent:

(1) $\mathbf{t} \in \llbracket A \rrbracket^{\mathbb{B}}(d)$

(2) $\mathbf{t} \in \llbracket A^{\mathbb{K}} \rrbracket^{\mathbb{K}}(d)$ and $\llbracket A^{\mathbb{K}} \rrbracket^{\mathbb{K}}(d)(\mathbf{t})=\overline{1}$.

Furthermore, $A^{\mathbb{K}}$ has exactly one run for every tuple in $\llbracket A^{\mathbb{K}} \rrbracket^{\mathbb{K}}(d)$.

We now show that a $\mathbb{B}$-projections of a $\mathbb{K}$-weighted VSet-automaton $A$ exists if $\mathbb{K}$ is positive. Furthermore, a $\mathbb{K}$-extensions of a $\mathbb{B}$-weighted VSet-automaton always exists. To this end, let $(\mathbb{K}, \oplus, \otimes, \overline{0}, \overline{1})$ and $\left(\mathbb{K}^{\prime}, \oplus^{\prime}, \otimes^{\prime}, \overline{0^{\prime}}, \overline{1^{\prime}}\right)$ be semirings. For a function $f: \mathbb{K} \rightarrow \mathbb{K}^{\prime}$ and a weighted VSet-automaton $A:=(\Sigma, V, Q, I, F, \delta)$ over $\mathbb{K}$, we define the weighted VSet-automaton $A_{f}:=\left(\Sigma, V, Q, I_{f}, F_{f}, \delta_{f}\right)$ over $\mathbb{K}^{\prime}$, where $I_{f}:=f \circ I, F_{f}:=f \circ F$, and $\delta_{f}:=f \circ \delta$.

Lemma 6.16. Let $\mathbb{K}$ be a positive semiring. Then there exists a $\mathbb{B}$-projection $A^{\mathbb{B}}$ of $A$ for every $\mathbb{K}$-weighted VSet-automaton A.

Proof. Let $f: \mathbb{K} \rightarrow \mathbb{B}$ be the function

$$
f(x)= \begin{cases}\text { true } & \text { if } x \neq \overline{0} \\ \text { false } & \text { if } x=\overline{0}\end{cases}
$$


Eilenberg [Eil74, Chapter VI.2] showed that, due to $\mathbb{K}$ being positive ${ }^{22}$, the function $f$ is a semiring morphism, that is,

$$
\begin{array}{ll}
f\left(x \oplus^{\mathbb{K}} y\right)=f(x) \oplus^{\mathbb{B}} f(y), & f(\overline{0})=\text { false } \\
f\left(x \otimes^{\mathbb{K}} y\right)=f(x) \otimes^{\mathbb{B}} f(y), & f(\overline{1})=\text { true. }
\end{array}
$$

Observe that these properties ensure that, for every document $d \in$ Docs and every tuple $\mathbf{t} \in \llbracket A \rrbracket^{\mathbb{K}}$, it holds that

$$
f\left(\llbracket A \rrbracket^{\mathbb{K}}(d)(\mathbf{t})\right)=\llbracket A_{f} \rrbracket^{\mathbb{K}^{\prime}}(d)(\mathbf{t}) .
$$

Therefore, $A_{f}$ is a $\mathbb{B}$-projection of $A$.

Lemma 6.17. Every $\mathbb{B}$-weighted VSet-automaton $A$ has a $\mathbb{K}$-extension.

Proof. Let $A:=(V, Q, I, F, \delta)$ be a $\mathbb{B}$-weighted VSet-automaton. Doleschal et al. $\left[\mathrm{DKM}^{+} 19\right.$, Proposition 4.4] showed that, for every VSet-automaton $A$, there is an equivalent deterministic VSet-automaton $A_{\text {det }}$. Note that a deterministic VSet-automaton has exactly one run for every tuple $\mathbf{t} \in \llbracket A_{\operatorname{det}} \rrbracket^{\mathbb{B}}(d)$. Therefore, w.l.o.g., we can assume that $A$ has this property. Let $g: \mathbb{B} \rightarrow \mathbb{K}$ be the function ${ }^{23}$

$$
g(x)= \begin{cases}\overline{1} & \text { if } x=\text { true } \\ \overline{0} & \text { if } x=\text { false }\end{cases}
$$

Observe that $A_{g}$ also has exactly one run for every tuple $\mathbf{t} \in \llbracket A_{\mathrm{det}} \rrbracket^{\mathbb{K}}(d)$. It remains to show that $A_{g}$ is indeed a $\mathbb{K}$-extension of $A$. To this end, let $d \in$ Docs be a document. We now show the equivalence between (1) and (2).

(1) implies (2): Let $\mathbf{t} \in \llbracket A \rrbracket^{\mathbb{B}}(d)$. Per assumption, $A$ has exactly one run $\rho$ on $d$ for $\mathbf{t}$. Let $\rho_{g}:=g(\rho)$ be the run, resulting from $\rho$ by replacing all weights $w$ with $g(w)$. Observe that $\rho_{g}$ must be a run of $A_{g}$ on $d$ accepting $\mathbf{t}$. Per construction, all transitions of $A_{g}$ have weight $\overline{0}$ or $\overline{1}$. Thus, (2) must hold.

(2) implies (1): Let $\mathbf{t} \in \llbracket A_{g} \rrbracket^{\mathbb{K}}(d)$ and $\llbracket A^{\prime} \rrbracket^{\mathbb{K}}(d)(\mathbf{t})=\overline{1}$. Thus, there is a run $\rho_{g}$ of $A_{g}$ on $d$ accepting $\mathbf{t}$. Therefore, there must also be a run $\rho$ of $A$ on $d$, accepting $\mathbf{t}$, concluding the proof.

We are now ready to prove the two main results of this section.

Lemma 6.18. Let $R$ be a string relation, which is selectable by document spanners. Then $R$ is also selectable by $\mathbb{K}$-Annotators.

Proof. Let $A$ be a $\mathbb{K}$-weighted VSet-automaton and $R$ be a relation that is selectable by regular $\mathbb{B}$-annotators. We have to show that every string selection $\sigma_{x_{1}, \ldots, x_{k}}^{R} \llbracket A \rrbracket^{\mathbb{K}}$ is definable by a $\mathbb{K}$-weighted VSet-automaton. By assumption $R$ is selectable by regular $\mathbb{B}$-annotators. Let $A_{R}^{\mathbb{B}}$ be the VSet-automaton that selects $R$ over $\mathbb{B}$, which exists by Lemma 6.13 . Let $A_{R}^{\mathbb{K}}$ be a $\mathbb{K}$-extension of $A_{R}^{\mathbb{B}}$ VSet-automaton, which exists by Lemma 6.17 . Thus, $A_{R}^{\mathbb{K}}$ selects $R$ over $\mathbb{K}$ and therefore, by Lemma $6.13, R$ is selectable by $\mathbb{K}$-Annotators.

Lemma 6.19. Let $(\mathbb{K}, \oplus, \otimes, \overline{0}, \overline{1})$ be a positive semiring and $R$ be a string relation, which is selectable by $\mathbb{K}$-Annotators. Then $R$ is also selectable by document spanners.

\footnotetext{
${ }^{22}$ Eilenberg [Eil74, Chapter VI.2] actually showed that $f$ is a semiring morphism if and only if $\mathbb{K}$ is positive.

${ }^{23}$ Notice that $g$ is not necessarily a semiring morphism. Depending on $\mathbb{K}$, it may be the case that $\overline{1} \oplus \overline{1}=\overline{0}$, contradicting the properties of semiring morphisms. Take $\mathbb{K}=\mathbb{Z} / 2 \mathbb{Z}$, for instance.
} 
Proof. Let $R$ be a string relation selectable by $\mathbb{K}$-annotators and let $A$ be a $\mathbb{B}$-weighted VSet-automaton. We have to show that $R$ is also selectable over $\mathbb{B}$, i.e., there is a $\mathbb{B}$ weighted VSet-automaton $A_{R}^{\mathbb{B}}$ such that $\llbracket A_{R}^{\mathbb{B}} \rrbracket^{\mathbb{B}}=\sigma_{x_{1}, \ldots, x_{k}}^{R} \llbracket A \rrbracket^{\mathbb{B}}$. Let $A^{\mathbb{K}}$ be a $\mathbb{K}$-extension of $A$, which exists by Lemma 6.17. Per assumption $R$ is selectable over $\mathbb{K}$, therefore, due to Lemma 6.13 , there exists a $\mathbb{K}$-weighted VSet-automaton $A_{R}^{\mathbb{K}}$ which selects $R$ over $\mathbb{K}$. Thus, $\sigma_{x_{1}, \ldots, x_{k}}^{R} \llbracket A^{\mathbb{K}} \rrbracket^{\mathbb{K}}=\llbracket A_{R}^{\mathbb{K}} \rrbracket^{\mathbb{K}}$. Let $A_{R}^{\mathbb{B}}$ be a $\mathbb{B}$-projection of $A_{R}^{\mathbb{K}}$, which exists by Lemma 6.16. It remains to show that $\sigma_{x_{1} \ldots, x_{k}}^{R} \llbracket A \rrbracket^{\mathbb{B}}=\llbracket A_{R}^{\mathbb{B}} \rrbracket^{\mathbb{B}}$. Let $\mathbf{t} \in \llbracket A_{R}^{\mathbb{B}} \rrbracket^{\mathbb{B}}$. By Lemma 6.16, $\mathbf{t} \in \llbracket A_{R}^{\mathbb{K}} \rrbracket^{\mathbb{K}}$ and therefore, $\mathbf{t} \in \sigma_{x_{1}, \ldots, x_{k}}^{R} \llbracket A^{\mathbb{K}} \rrbracket^{\mathbb{K}}$. Per definition of string selection, it follows that $\left(d_{\mathbf{t}\left(x_{1}\right)}, \ldots, d_{\mathbf{t}\left(x_{k}\right)}\right) \in R$ and $\mathbf{t} \in \llbracket A_{\mathbb{K}} \rrbracket^{\mathbb{K}}$. By Lemma 6.17 , it follows that $\left(d_{\mathbf{t}\left(x_{1}\right)}, \ldots, d_{\mathbf{t}\left(x_{k}\right)}\right) \in R$ and $\mathbf{t} \in \llbracket A \rrbracket^{\mathbb{B}}$, and therefore $\mathbf{t} \in \sigma_{x_{1}, \ldots, x_{k}}^{R} \llbracket A \rrbracket^{\mathbb{B}}$. Observe that all implications in the previous argument where actually equivalences. Therefore, the inclusion $\sigma^{R} \llbracket A \rrbracket^{\mathbb{B}} \subseteq \llbracket A_{R}^{\mathbb{B}} \rrbracket^{\mathbb{B}}$ also holds.

Observe that for the proof of Theorem 6.11 we only required positivity of the semiring for the implication from (3) to (2). This raises the question whether the equivalence can be generalized even further. We show next that this is indeed the case, such as for the Łukasiewicz semiring, which is not positive.

6.4.1. Beyond Positive Semirings. We provide some insights about the cases where $\mathbb{K}$ is not positive. Recall that, by Lemma 6.18 , every string relation $R$, which is selectable by document spanners is also selectable by $\mathbb{K}$-Annotators. Therefore, the question is: for which semirings $\mathbb{K}$ does selectability by $\mathbb{K}$-annotators imply selectability by ordinary document spanners? It turns out that this is indeed possible for some non-positive semirings, such as the Eukasiewicz semiring $\mathrm{E}$.

Let $\left(\mathbb{K}^{\prime}, \oplus^{\prime}, \otimes^{\prime}, \overline{0^{\prime}}, \overline{1^{\prime}}\right)$ be a subsemiring of a semiring $\mathbb{K}^{24}$ This semiring is minimal if there is no subsemiring of $(\mathbb{K}, \oplus, \otimes, \overline{0}, \overline{1})$ with fewer elements. Recall that a semiring $\mathbb{K}$ is bipotent, if $a \oplus b \in\{a, b\}$, for every $a, b \in \mathbb{K}$. We begin with some intermediate results.

Lemma 6.20. Let $(\mathbb{K}, \oplus, \otimes, \overline{0}, \overline{1})$ be a bipotent semiring. Then $\mathbb{K}_{\min }:=\{\overline{0}, \overline{1}\}$ is the unique minimal subsemiring of $\mathbb{K}$. Furthermore, $\mathbb{K}_{\min }$ is isomorphic to the Boolen semiring.

Proof. For every semiring it holds that $\overline{0} \otimes \overline{1}=\overline{0}, \overline{1} \otimes \overline{0}=\overline{0}$, and $\overline{0} \otimes \overline{0}=\overline{0}$. Furthermore, $\overline{1} \oplus \overline{0}=\overline{1}$ and $\overline{0} \oplus \overline{0}=\overline{0}$. As $\mathbb{K}$ is bipotent, it also holds that $\overline{1} \oplus \overline{1}=\overline{1}$. Recall that a subsemiring of $\mathbb{K}$ is a set $\mathbb{K}^{\prime}$, closed under addition and multiplication. Let $\mathbb{K}^{\prime}=\{\overline{0}, \overline{1}\}$. Thus, $\mathbb{K}_{\text {min }}=\{\overline{0}, \overline{1}\}$ is a subsemiring of $\mathbb{K}$, as $\{\overline{0}, \overline{1}\}$ is closed under addition and multiplication. Observe that $\mathbb{K}_{\text {min }}$ must be unique and minimal, as every subsemiring must at least contain $\overline{0}$ and $\overline{1}$.

It remains to show that $\mathbb{K}_{\text {min }}$ is isomorphic to $\mathbb{B}$. To this end, let $f: \mathbb{K}_{\min } \rightarrow \mathbb{B}$ be the bijection

$$
f(x)= \begin{cases}\text { true } & \text { if } x=\overline{1} \\ \text { false } & \text { if } x=\overline{0}\end{cases}
$$

It is straightforward to verify that $f$ is indeed a semiring isomorphism.

It follows directly that $\mathbb{K}_{\min }$ is a positive semiring.

\footnotetext{
${ }^{24}$ Recall that a subsemiring of $\mathbb{K}$ is a set $\mathbb{K}^{\prime}$, closed under addition and multiplication.
} 
Lemma 6.21. Let $\mathbb{K}$ be a bipotent semiring such that $a \otimes b=\overline{1}$ implies that $a=b=\overline{1}$. Then a string relation $R$ is selectable by $\mathbb{K}$-annotators if and only if it is selectable by $\mathbb{K}_{\text {min }}$-annotators.

Proof. Every $\mathbb{K}_{\mathrm{min}}$-annotator is also a $\mathbb{K}$-annotator. Therefore, we only have to show that every string relation selectable by $\mathbb{K}$-annotators is also selectable by $\mathbb{K}_{\text {min }}$-annotators.

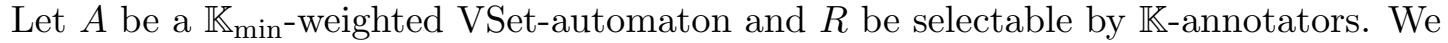
have to show that $\sigma_{x_{1}, \ldots, x_{k}}^{R} \llbracket A \rrbracket^{\mathbb{K}_{\min }}$ is definable by a $\mathbb{K}_{\text {min }}$-weighted VSet-automaton. Let $d \in$ Docs be a document.

Per assumption $R$ is selectable over $\mathbb{K}$. Let $A_{\mathbb{K}}^{R}$ be a $\mathbb{K}$-weighted VSet-automaton, guaranteed by Lemma 6.13 . Therefore, $A_{\mathbb{K}}^{R}$ only assigns weight $\overline{1}$ and $\overline{0}$. Recall that $\mathbb{K}$ is bipotent, that is, for every $a, b \in \mathbb{K}$, it holds that $a \oplus b \in\{a, b\}$. Therefore, for every $\mathbf{t} \in \llbracket A_{\mathbb{K}}^{R} \rrbracket^{\mathbb{K}}(d)$ there must be a run $\rho$ of $A_{\mathbb{K}}^{R}$ on $d$ with $\mathrm{w}_{\rho}=\overline{1}$. Furthermore, as $a \otimes b=\overline{1}$ implies that $a=b=\overline{1}$, this run must not have an edge with weight $a \neq \overline{1}$. The existence a run $\rho$ of $A_{\mathbb{K}}^{R}$ on $d$ with weight $\mathrm{w}_{\rho}=\overline{1}$ also implies that $\mathbf{t}_{\rho} \in \llbracket A_{\mathbb{K}}^{R} \rrbracket^{\mathbb{K}}(d)$. Thus, there is a run $\rho$ of $A_{\mathbb{K}}^{R}$ on $d$ consisting only of edges with weight $\overline{1}$ if and only if $\mathbf{t}_{\rho} \in \llbracket A_{\mathbb{K}}^{R} \rrbracket^{\mathbb{K}}$. Therefore, we can assume, w.l.o.g., that all edges in $A_{\mathbb{K}}^{R}$ have either weight $\overline{0}$ or $\overline{1}$. Thus, $A_{\mathbb{K}}^{R}$ is a

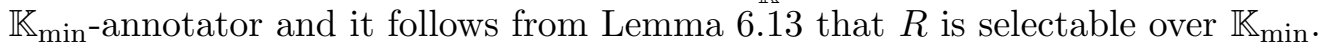

Corollary 6.22. Let $\mathbb{K}$ be a bipotent semiring, such that $a \otimes b=\overline{1}$ implies that $a=b=\overline{1}$. $A$ string relation $R$ is recognizable if and only if it is selectable by $\mathbb{K}$-annotators.

Proof. Follows directly from Theorems 6.11, 6.21, and Lemma 6.20.

Recall the Eukasiewcz semiring, whose domain is $[0,1]$, with addition given by $x \oplus y=$ $\max (x, y)$, with multiplication $x \otimes y=\max (0, x+y-1)$, zero element 0 , and one element 1. Thus, for every $a, b \in[0,1], a \oplus b \in\{a, b\}$ and $a \otimes b=1$ if and only if $a=b=1$. Therefore, the Łukasiewcz semiring satisfies the conditions of Corollary 6.22.

Corollary 6.23. Let $E$ be the Lukasiewcz semiring. A string relation $R$ is recognizable if and only if it is selectable by E-annotators.

\section{Evaluation Problems}

We consider two types of evaluation problems in this section: answer testing and best weight evaluation. The former is given an annotator, document $d$, and tuple $\mathbf{t}$, and computes the annotation of $\mathbf{t}$ in $d$ according to the annotator. The latter does not receive the tuple as input, but receives a weight threshold and is asked whether there exists a tuple to which a weight greater than or equal to the threshold is assigned.

7.1. Answer Testing. It follows from Freydenberger [Fre19, Lemma 3.1] that answer testing is NP-complete for $\mathbb{B}$-weighted VSet-automata in general. Indeed, he showed that, given a $\mathbb{B}$-weighted VSet-automaton $A$, it is NP-complete to check if $A$ returns an output on the empty document, so it is even NP-complete to check if the tuple of empty spans is returned or not. However, the proof makes extensive use of non-functionality of the automaton. Indeed, we can prove that answer testing is tractable for functional weighted VSet-automata.

Theorem 7.1. Given a functional weighted VSet-automaton A, a document d, and a tuple $\mathbf{t}$, the weight $\llbracket A \rrbracket^{\mathbb{K}}(d)(\mathbf{t})$ assigned to $\mathbf{t}$ by $A$ on $d$ can be computed in polynomial time. 
Proof. Let $A, d$, and $\mathbf{t}$ be as stated. Per definition, the weight assigned to $\mathbf{t}$ by $A$ is

$$
\llbracket A \rrbracket^{\mathbb{K}}(d)(\mathbf{t}):=\bigoplus_{\rho \in \operatorname{Runs}(A, d) \text { and } \mathbf{t}=\mathbf{t}_{\rho}} \mathrm{w}_{\rho} .
$$

Therefore, in order to compute the weight $\llbracket A \rrbracket^{\mathbb{K}}(d)(\mathbf{t})$, we need to consider the weights of all runs $\rho$ for which $\mathbf{t}=\mathbf{t}_{\rho}$. Furthermore, multiple runs can select the same tuple $\mathbf{t}$ but assign variables in a different order. ${ }^{25}$

Let $A_{E}$ be the functional extended weighted VSet-automaton corresponding to $A$, as constructed by Proposition 6.7. It follows that $\llbracket A \rrbracket^{\mathbb{K}}(d)(\mathbf{t})=\llbracket A_{E} \rrbracket^{\mathbb{K}}(d)(\mathbf{t})$. Let $\rho$ be a run of $A_{E}$ on $d$ and $\mathbf{t}$, and let $d_{\mathbf{t}}$ be the document obtained from $\rho$ by concatenating the labels of the transitions of $\rho .^{26}$ Observe that $d_{\mathbf{t}}$ encodes $d$ and $\mathbf{t}$ and is uniquely defined by $d$ and $\mathbf{t}$.

It follows that, if $A_{E}$ is interpreted as an weighted automaton, the weight $\llbracket A_{E} \rrbracket^{\mathbb{K}}(d)(\mathbf{t})$ is exactly the weight which is assigned by $A_{E}$ to the input word $d_{\mathbf{t}}$.

We note that computing the weight assigned by a weighted automaton on an input word $w$ strongly depends on the cost model and the used semiring. We therefore give an explicit proof that the weight can be computed in polynomial time if the semiring has an efficient encoding.

We define an functional extended weighted VSet-automaton $A_{\mathbf{t}}$, such that $\llbracket A_{\mathbf{t}} \rrbracket^{\mathbb{K}}(d)(\mathbf{t})=$ $\overline{1}$ and $\llbracket A_{\mathbf{t}} \rrbracket^{\mathbb{K}}(d)\left(\mathbf{t}^{\prime}\right)=\overline{0}$ for all $\mathbf{t}^{\prime} \neq \mathbf{t}$. Such an automaton $A_{\mathbf{t}}$ can be defined using a chain of $\left|d_{\mathbf{t}}\right|+1$ states, which checks that the input document is $d$ and which has exactly one nonzero run $\rho$, with $\mathrm{w}_{\rho}=\overline{1}$ and $\mathbf{t}_{\rho}=\mathbf{t}$.

By Proposition 6.8, there is a weighted VSet-automaton $A^{\prime}$ such that $\llbracket A^{\prime} \rrbracket^{\mathbb{K}}=\llbracket A_{E} \rrbracket^{\mathbb{K}} \bowtie$ $\llbracket A_{\mathbf{t}} \rrbracket^{\mathbb{K}}$. It follows directly from the definition of $A^{\prime}$ that $\llbracket A^{\prime} \rrbracket^{\mathbb{K}}\left(d^{\prime}\right)\left(\mathbf{t}^{\prime}\right)=\overline{0}$ if $d^{\prime} \neq d$ or $\mathbf{t}^{\prime} \neq \mathbf{t}$ and $\llbracket A^{\prime} \rrbracket^{\mathbb{K}}(d)(\mathbf{t})=\llbracket A \rrbracket^{\mathbb{K}}(d)(\mathbf{t})$, otherwise. Furthermore, all runs $\rho \in$ Runs $\left(A^{\prime}, d\right)$ have length $\left|d_{\mathbf{t}}\right|+1$. Therefore, the weight $\llbracket A^{\prime} \rrbracket^{\mathbb{K}}(d)(\mathbf{t})$ can be obtained by taking the sum of the weights of all runs of length $\left|d_{\mathbf{t}}\right|+1$ of $A^{\prime}$. If we assume, w.l.o.g., that the states of $A^{\prime}$ are $\{1, \ldots, n\}$ for some $n \in \mathbb{N}$, then, due to distributivity of $\oplus$ over $\otimes$, this sum can be computed as

$$
\llbracket A^{\prime} \rrbracket^{\mathbb{K}}(d)(\mathbf{t})=v_{I} \times\left(M_{\delta}\right)^{\left|d_{\mathbf{t}}\right|+1} \times\left(v_{F}\right)^{T},
$$

where

- $v_{I}$ is the vector $(I(1), \ldots, I(n))$,

- $M_{\delta}$ is the $n \times n$ matrix with $M_{\delta}(i, j)=\bigoplus_{a \in \Sigma \cup \Gamma_{V}} \delta(i, a, j)$, and

- $\left(v_{F}\right)^{T}$ is the transpose of vector $v_{F}=(F(1), \ldots, F(n))$.

Therefore, by the assumption that $\mathbb{K}$ has an efficient encoding (Definition 5.1), the weight can be computed in polynomial time.

7.2. Best Weight Evaluation. In many semirings, the domain is naturally ordered by some relation. For instance, the domain of the probability semiring is $\mathbb{Q}^{+}$, which is ordered

\footnotetext{
${ }^{25}$ This may happen when variable operations occur consecutively, that is, without reading an alphabet symbol in between.

${ }^{26}$ We note that we assume in the following that every set $X \subseteq \Gamma_{V}$ is represented by an unique label that is not in $\Sigma$. That is, we can assume w.l.o.g. that no set of the form $X \subseteq \Gamma_{V}$ is an element from $\Sigma$ and just use subsets of $\Gamma_{V}$ as labels.
} 
by the $\leq$-relation. This motivates evaluation problems, where one is interested in some kind of optimization of the weight. We start by giving the definition of an ordered semiring. ${ }^{27}$

Definition 7.2 (similar to Droste and Kuich [DK09]). A commutative monoid $(\mathbb{K}, \oplus, \overline{0})$ is ordered if it is equipped with a linear order $\preccurlyeq$ preserved by the $\oplus$ operation. An ordered monoid is positively ordered if $\overline{0} \preccurlyeq a$ for all $a \in \mathbb{K}$. A semiring $(\mathbb{K}, \oplus, \otimes, \overline{0}, \overline{1})$ is (positively) ordered if the additive monoid is (positively) ordered and multiplication with elements $\overline{0} \preccurlyeq a$ preserves the order.

We consider the following two problems.

\section{THRESHOLD}

Given: Regular annotator $A$ over an ordered semiring, document $d \in$ Docs, and a weight $w \in \mathbb{K}$.

Question: Is there a tuple $\mathbf{t}$ with $w \preccurlyeq \llbracket A \rrbracket^{\mathbb{K}}(d)(\mathbf{t})$ ?

MAXTUPLE

Given: Regular annotator $A$ over an ordered semiring and a document $d \in$ Docs.

Task: Compute a tuple with maximal weight, if it exists.

Notice that, if MaxTuple is efficiently solvable, then so is Threshold. We therefore prove upper bounds for MAXTuple and lower bounds for Threshold. The Threshold problem is sometimes also called the emptiness problem in the weighted automata literature. It turns out that both problems are tractable for positively ordered semirings that are bipotent (that is, for every $a, b \in \mathbb{K}$ it holds that $a \oplus b \in\{a, b\}$ ).

We first make an observation about positively ordered, bipotent semirings.

Observation 7.3. Let $(\mathbb{K}, \oplus, \otimes, \overline{0}, \overline{1})$ be a positively ordered, bipotent semiring. Then, for every $a, b \in \mathbb{K}$ with $a \preccurlyeq b$ it holds that $a \oplus b=b$.

Proof. As $\mathbb{K}$ is positively ordered it holds that $\overline{0} \preccurlyeq a$. Thus,

$$
\overline{0} \oplus b \preccurlyeq a \oplus b,
$$

and therefore $b \preccurlyeq a \oplus b$. Due to $\mathbb{K}$ being bipotent, $a \oplus b \in\{a, b\}$. Assume that $a \oplus b=a$. Thus, $b \preccurlyeq a$ and it follows with $a \preccurlyeq b$ and antisymmetry of $\preccurlyeq$ that $a=b$. Therefore, $a \oplus b=b$ as claimed.

Notice that Observation 7.3 shows that, for positively ordered, bipotent semirings, the $\oplus$-operator is the same as the max operator, where the maximum is taken over the linear order $\preccurlyeq$.

Corollary 7.4. Let $(\mathbb{K}, \oplus, \otimes, \overline{0}, \overline{1})$ be a positively ordered, bipotent semiring. Then $\oplus=\max$.

We prove our tractability result for positively ordered, bipotent semirings. Due to Corollary 7.4, we can assume that $\oplus$ is the maximum operator over $\preccurlyeq$.

\footnotetext{
${ }^{27}$ Note that the following definition slightly deviates from the definition by Droste and Kuich [DK09]. We require the order to be linear, as the maximal weight would otherwise not be well defined.
} 
Theorem 7.5. Let $(\mathbb{K}, \oplus, \otimes, \overline{0}, \overline{1})$ be a positively ordered semiring, where $\oplus=\max$ is the maximum operator over the linear order $\preccurlyeq$. Furthermore, let $A$ be a functional $\mathbb{K}$-weighted $V$ Set-automaton, and let $d \in$ Docs be a document. Then MAXTuple for $A$ and $d$ can be solved in polynomial time.

Proof. By Proposition 6.7, we can assume, w.l.o.g., that $A$ is given as an extended functional $\mathbb{K}$-weighted VSet-automaton. Furthermore, as $\mathbb{K}$ is bipotent, it must hold that $a \oplus b \in\{a, b\}$ for every $a, b \in \mathbb{K}$. Therefore, the weight of a tuple $t \in \llbracket A \rrbracket^{\mathbb{K}}(d)$ is always equal to the weight of one of the runs $\rho$ with $\mathbf{t}=\mathbf{t}_{\rho}$.

Let $\rho \in \operatorname{Runs}(A, d)$ be the run of $A$ on $d$ with maximal weight. Due to $\oplus=\max$, it must hold that $\mathbf{w}_{\mathbf{t}_{\rho}}=\mathrm{w}_{\rho}$ and $\mathrm{w}_{\mathbf{t}} \preccurlyeq \mathrm{w}_{\mathbf{t}_{\rho}}$, as otherwise, $\rho$ would not be the run of $A$ on $d$ with maximal weight. Therefore, in order to find the tuple with maximal weight, we need to find the run of $A$ on $d$ with maximal weight.

To this end, we define a directed acyclic graph (DAG) which is obtained by taking a "product" between $A$ and $d$. Finding the run with the maximal weight then boils down to finding the path with maximal weight in this DAG.

Assume that $A=(V, Q, I, F, \delta)$. Recall that $2^{\Gamma_{V}}$ denotes the power set of $\Gamma_{V}$. We define a weighted, edge-labeled DAG $G=(N, E, w)$, where each edge $e$ is in $N \times\left(\{\varepsilon\} \uplus\left(2^{\Gamma_{V}} \times\right.\right.$ $\{1, \ldots,|d|+1\})) \times N$ and $w$ assigns a weight $w(e) \in \mathbb{K}$ to every edge $e$. We note that an edge $(p,(T, i), q) \in E$ will encodes that a transition, labeled $T$, is reached after reading $d_{[1, i\rangle}$.

More formally, let $N:=\{s, t\} \uplus\{(q, i) \mid q \in Q$ and $1 \leq i \leq|d|+1\}$. We say that a node $n=(p, i)$ is in layer $i$ of $G$, where $s$ is in layer 0 and $t$ in layer $|d|+2$. Furthermore, let $E$ be defined as follows:

$$
\begin{aligned}
E & :=\{(s, \varepsilon,(q, 1)) \mid I(q) \neq \overline{0}\} \\
& \cup\{((q,|d|+1), \varepsilon, t) \mid F(q) \neq \overline{0}\} \\
& \cup\left\{((p, i),(T, i),(q, i)) \mid T \subseteq \Gamma_{V} \text { and } \delta(p, T, q) \neq \overline{0}\right\} \\
& \cup\left\{((p, i), \varepsilon,(q, i+1)) \mid d_{[i, i+1\rangle}=a \text { and } \delta(p, a, q) \neq \overline{0}\right\} .
\end{aligned}
$$

Furthermore, for $T \subseteq \Gamma_{V}$ and $a \in \Sigma$, we define the weight $w(e)$ for all $e \in E$ as follows:

$$
\begin{aligned}
w((s, \varepsilon,(q, 1))) & :=I(q) \\
w(((q,|d|+1), \varepsilon, t)) & :=F(q) \\
w(((p, i),(T, i),(q, i))) & :=\delta(p, T, q) \\
w(((p, i), \varepsilon,(q, i+1))) & :=\delta(p, a, q) .
\end{aligned}
$$

Recall that, in extended weighted VSet-automata, the set of states $Q$ is a disjoint union of letter- and variable states, such that all transitions labeled by $\sigma \in \Sigma$ originate in letter states and all transitions labeled by $T \subseteq \Gamma_{V}$ originate in variable states. Therefore, $G$ must be acyclic, as all edges are either from a node in layer $i$ to a node in layer $i+1$ or from a variable state to a letter state within the same layer. Furthermore, there is a path from $s$ to $t$ in $G$ with weight $w$ if and only if there is a tuple $\mathbf{t} \in \llbracket A \rrbracket^{\mathbb{K}}(d)$ with the same weight.

As shown in Mohri [Moh09], a path of maximal weight can be computed in polynomial time. We also give procedure, Procedure BestWeightEvaluation, for the sake of succinctness. ${ }^{28}$ The correctness follows directly from $\mathbb{K}$ being positively ordered, thus order being preserved by addition and multiplication with an element $\ell \in \mathbb{K}$.

\footnotetext{
${ }^{28}$ We note that all semiring operations must be computable efficiently as we assume that only efficient encodings are used.
} 


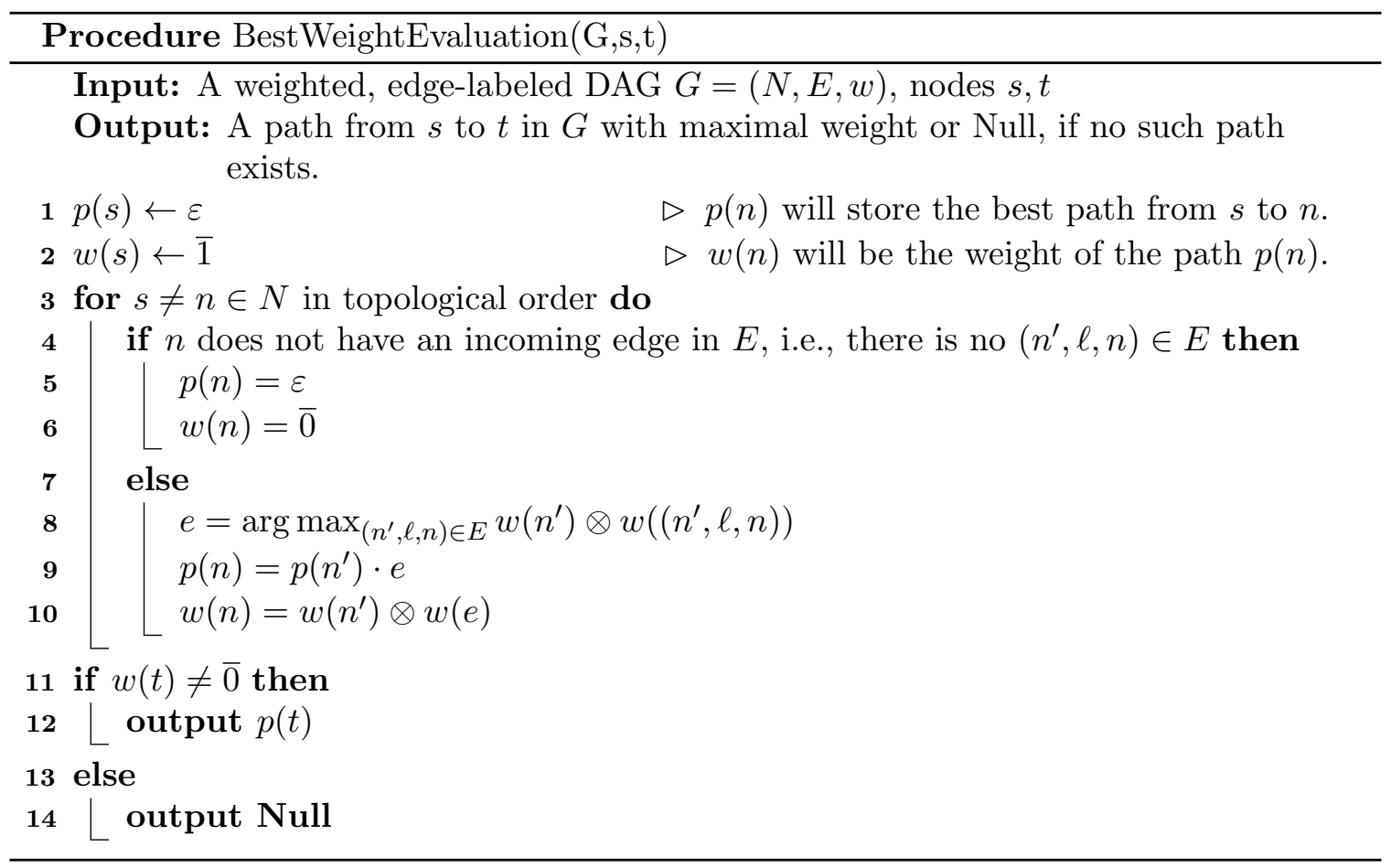

If the semiring is not bipotent, however, the THRESHOLD and MAXTUPLE problems quickly become intractable.

Theorem 7.6. Let $(\mathbb{K}, \oplus, \otimes, \overline{0}, \overline{1})$ be a semiring such that $\bigoplus_{i=1}^{m} \overline{1}$ is strictly monotonously increasing for increasing values of $m$. Furthermore, let $A$ be a functional $\mathbb{K}$-weighted VSetautomaton, let $d \in$ Docs be a document, and $k \in \mathbb{K}$ be a weight threshold. Then THREsHOLD for such inputs is NP-complete.

Proof. It is obvious that THRESHOLD is in NP, as one can guess a tuple $\mathbf{t}$ and test in PTIME whether $w \preccurlyeq \llbracket A \rrbracket^{\mathbb{K}}(d)(\mathbf{t})$ using Theorem 7.1.

For the NP-hardness, we will reduce from the MAX-3SAT problem. Given a 3CNF formula and a natural number $k$, the decision version of MAX-3SAT asks whether there is a valuation satisfying at least $k$ clauses. Let $\psi=C_{1} \wedge \cdots \wedge C_{m}$ be a Boolean formula in $3 \mathrm{CNF}$ over variables $x_{1}, \ldots, x_{n}$ such that each clause $C_{i}=\left(\ell_{i, 1} \vee \ell_{i, 2} \vee \ell_{i, 3}\right)$ is a disjunction of exactly three literals $\ell_{i, j} \in\left\{x_{c}, \neg x_{c} \mid 1 \leq c \leq n\right\}, 1 \leq i \leq k, 1 \leq j \leq 3$. We can assume, w.l.o.g., that no clause has two literals corresponding to the same variable. Observe that, for each clause $C_{i}$, there are $2^{3}=8$ assignments of the variables corresponding to the literals of $C_{i}$ of which exactly 7 satisfy the clause $C_{i}$. Formally, let $f_{C_{i}}$ be the function that maps a variable assignment $\tau$ to a number between 1 and 8 , depending on the assignments of the literals of the clause $C_{i}$. We assume, w.l.o.g., that $f_{C_{i}}(\tau)=8$ if and only if $C_{i}$ is not satisfied by $\tau$.

We define a functional weighted automaton automaton $A_{\psi}$ over the unary alphabet $\Sigma=\{\sigma\}$ such that $\llbracket A_{\psi} \rrbracket^{\mathbb{K}}\left(\sigma^{n}\right)(\mathbf{t})=\bigoplus_{i=1}^{m} \overline{1}$ if and only if the assignment corresponding to t satisfies exactly $m$ clauses in $\psi$ and $\llbracket A_{\psi} \rrbracket^{\mathbb{K}}(d)(\mathbf{t})=\overline{0}$ if $d \neq \sigma^{n}$ or $\mathbf{t}$ does not encode a variable assignment. To this end, each variable $x_{i}$ of $\psi$ is associated with a corresponding 


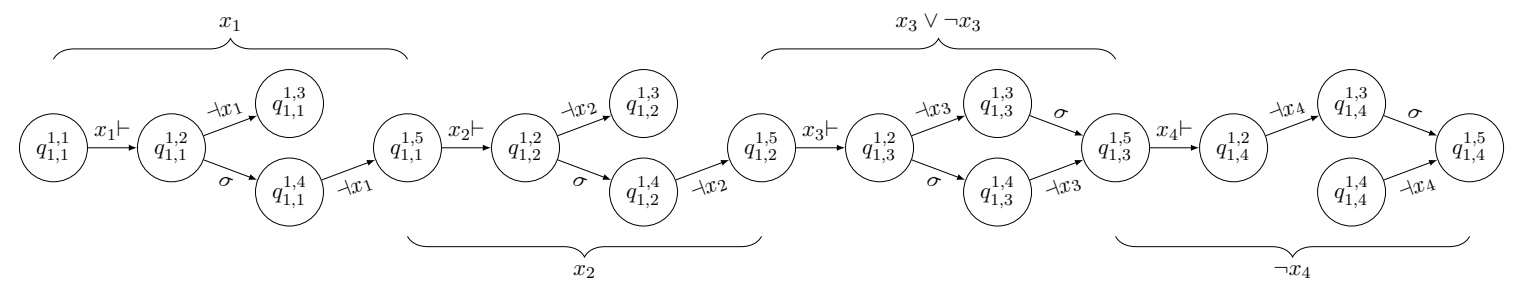

Figure 3: The sub-branch of $A_{\psi}$ corresponding to $C_{1}$ and $x_{1}=x_{2}=1, x_{4}=0$.
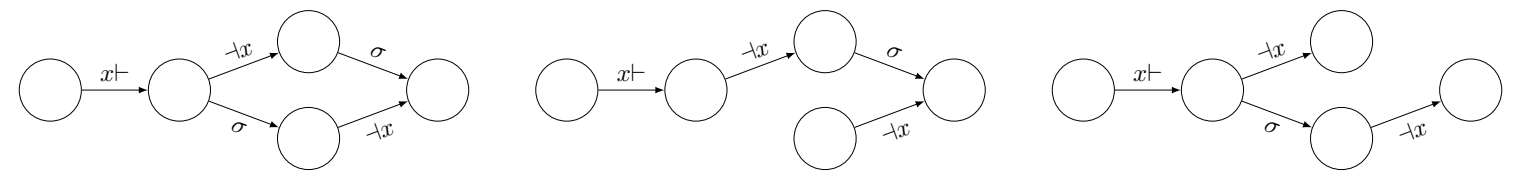

Figure 4: Example gadgets for variable $x$.

capture variable $x_{i}$ of $A_{\psi}$. With each assignment $\tau$ we associate a tuple $\mathbf{t}_{\tau}$, such that

$$
\mathbf{t}_{\tau}\left(x_{i}\right)= \begin{cases}{[i, i\rangle} & \text { if } \tau\left(x_{i}\right)=0, \text { and } \\ {[i, i+1\rangle} & \text { if } \tau\left(x_{i}\right)=1 .\end{cases}
$$

The automaton $A_{\psi}:=(\Sigma, V, Q, I, F, \delta)$ consists of $m$ disjoint branches, where each branch corresponds to a clause of $\psi$; we call these clause branches. Each clause branch is divided into 7 sub-branches, such that a path in the sub-branch $j$ corresponds to a variable assignment $\tau$ if $f_{C_{i}}(\tau)=j$. Thus, each clause branch has exactly one run $\rho$ with weight $\overline{1}$ for each tuple $\mathbf{t}_{\tau}$ associated to a satisfying assignment $\tau$ of $C_{i}$.

More formally, the set of states $Q=\left\{q_{i, j}^{a, b} \mid 1 \leq i \leq m, 1 \leq j \leq n, 1 \leq a \leq 7,1 \leq b \leq 5\right\}$ contains $5 n$ states for every of the 7 sub-branches of each clause branch. Intuitively, $A_{\psi}$ has a gadget, consisting of 5 states, for each variable and each of the 7 satisfying assignments of each clause. Figure 4 depicts the three types of gadgets we use here. Note that the weights of the drawn edges are all $\overline{1}$. We use the left gadget if $x$ does not occur in the relevant clause and the middle (resp., right) gadget if the literal $\neg x$ (resp., $x$ ) occurs. Furthermore, within the same sub-branch of $A_{\psi}$, the last state of each gadget is the same state as the start state of the next variable, i.e., $q_{i, j}^{a, 5}=q_{i, j+1}^{a, 1}$ for all $1 \leq i \leq k, 1 \leq j<n, 1 \leq a \leq 7$.

We illustrate the crucial part of the construction on an example. Let $\psi=\left(x_{1} \vee \neg x_{2} \vee\right.$ $\left.x_{4}\right) \wedge\left(x_{2} \vee x_{3} \vee x_{4}\right)$. The corresponding weighted VSet-automaton $A_{\psi}$ therefore has $14=2 \times 7$ disjoint branches. Figure 3 depicts the sub-branch for clause $C_{1}$ that corresponds to all assignments with $x_{1}=x_{2}=1$ and $x_{4}=0$.

Formally, the initial weight function is $I\left(q_{i, j}^{a, b}\right)=\overline{1}$ if $j=1=b$ and $I\left(q_{i, j}^{a, b}\right)=\overline{0}$ otherwise. The final weight function $F\left(q_{i, j}^{a, b}\right)=\overline{1}$ if $j=n$ and $b=5$ and $F\left(q_{i, j}^{a, b}\right)=\overline{0}$, otherwise. The 
transition function $\delta$ is defined as follows:

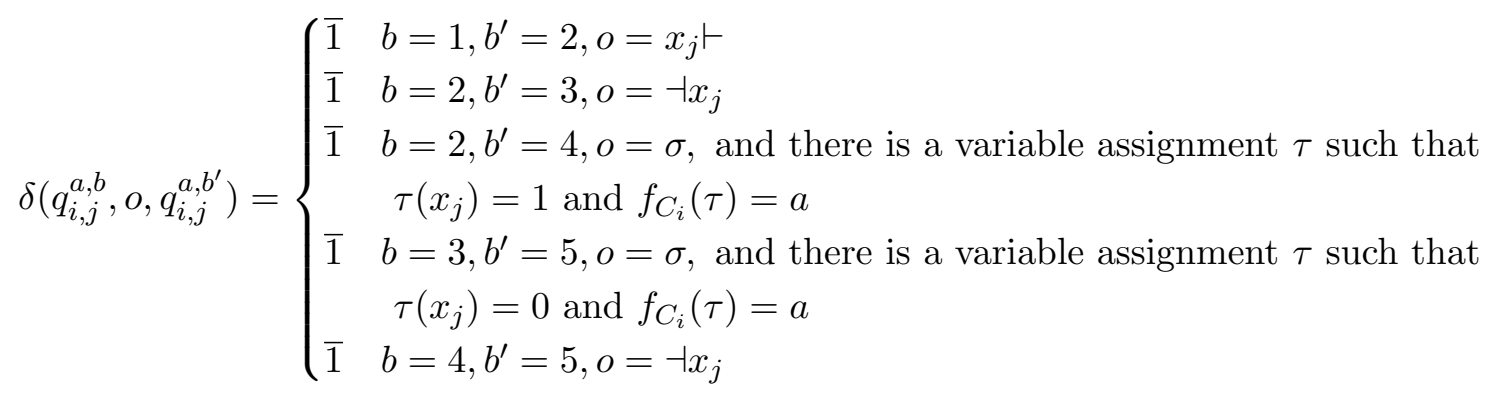

All other transitions have weight $\overline{0}$.

We show that there is a tuple $\mathbf{t} \in \llbracket A_{\psi} \rrbracket^{\mathbb{K}}\left(\sigma^{n}\right)$ with weight $\mathbf{w}_{\mathbf{t}}=\bigoplus_{i=1}^{k} \overline{1}$ if and only if the corresponding assignment $\tau$ satisfies exactly $k$ clauses of $\psi$. To this end, let $\tau$ be an assignment of the variables $x_{1}, \ldots, x_{n}$. Thus, there is a run $\rho \in \operatorname{Runs}\left(A_{\psi}, \sigma^{n}\right)$ with weight $\mathrm{w}_{\rho}=\overline{1}$ starting in $q_{i, 0}^{a, 0}$, such that $a=f_{C_{i}}(\tau)$ if and only if $\tau$ satisfies clause $C_{i}$. Due to $\bigoplus_{i=1}^{k} \overline{1}$ being strictly monotonously increasing it follows that $\bigoplus_{i=1}^{k} \overline{1} \preccurlyeq \mathbf{w}_{\mathbf{t}_{\tau}}$ if and only if the corresponding assignment to $\tau$ satisfies at least $k$ clauses. Let $w=\bigoplus_{i=1}^{k} \overline{1}$. It follows directly that there is an assignment $\tau$ of $\psi$ satisfying $k$ clauses if and only if there is a tuple t with $w \preccurlyeq \llbracket A_{\psi} \rrbracket^{\mathbb{K}}\left(\sigma^{n}\right)(\mathbf{t})$.

We note that Theorem 7.5 and Theorem 7.6 give us tight bounds for all semirings we defined in Example 2.1.

Since MAX-3SAT is hard to approximate, we can turn Theorem 7.6 into an even stronger inapproximability result for semirings where approximation makes sense. To this end, we focus on semirings that contain $(\mathbb{N},+, \cdot, 0,1)$ as a subsemiring in the following result. Note that this already implies that $\bigoplus_{i=1}^{m} \overline{1}$ is strictly monotonously increasing for increasing values of $m$.

Theorem 7.7. Let $\mathbb{K}$ be a semiring that contains $(\mathbb{N},+, \cdot, 0,1)$ as a subsemiring and let $A$ be a weighted VSet-automaton over $\mathbb{K}$. Unless PTIME $=N P$, there is no algorithm that approximates the tuple with the best weight within a sub-exponential factor in polynomial time.

Proof. Given a Boolean formula $\psi$ in 3CNF, MAX-3SAT asks for the maximal number of clauses satisfied by a variable valuation. Given a $3 \mathrm{CNF}$ formula, a polynomial time approximation algorithm with approximation factor $x$ returns a variable assignment satisfying at least $\frac{\mathrm{opt}}{x}$ clauses, where opt is the maximal number of clauses which are satisfiable by a single variable assignment. Håstad [Hås01] showed that, for every $\varepsilon>0$, it is NP-hard to approximate MAX-3SAT with an approximation factor $x<8 / 7-\varepsilon$, even if the input is restricted to satisfiable $3 \mathrm{CNF}$ instances. In other words, unless PTIME $=\mathrm{NP}$, for every polynomial time approximation algorithm, there is a satisfiable $3 \mathrm{CNF}$ instance such that, for this instance, the algorithm does not return an approximation with approximation factor $x<8 / 7-\varepsilon$. We can leverage this, using the reduction from Theorem 7.6 , to show that there is no polynomial time algorithm that approximates the tuple with the best weight with a sub-exponential approximation factor.

Let $\psi$ be a satisfiable 3CNF formula with $m$ clauses and let $A_{\psi}$ be the weighted VSetautomaton and $d \in$ Docs be as constructed from $\psi$ in the proof of Theorem 7.6. Let $c$ be the size of $A_{\psi}$, which is linear in $n$. As shown in Theorem 7.6, there is a tuple $\mathbf{t}$ in $A_{\psi}$ 
with weight $j$ if and only if the variable assignment corresponding to $\mathbf{t}$ satisfies exactly $j$ clauses. For a $k \in \mathbb{N}$ let $A_{\psi}^{k}$ be the weighted VSet-automaton, constructed by concatenating $k$ fresh copies of $A_{\psi}$, each of which operates on a set of $n$ fresh variables, by inserting $\varepsilon$-edges with weight $\overline{1}$ from $q_{i}$ to $q_{i+1}$ where $q_{i}$ is a final state of the $i$-th copy and $q_{i+1}$ an initial state of the $i+1$-th copy. Observe that $A_{\psi}^{k}$ has size $c \cdot k$, has $n k$ variables, and each tuple $\mathbf{t} \in \llbracket A_{\psi}^{k} \rrbracket^{\mathbb{K}}\left(d^{k}\right)$ encodes $k$, possibly different, variable assignments for $\psi$.

For the sake of contradiction, assume there is a polynomial time algorithm approximating the tuple with the best weight within a sub-exponential factor $f(x) \in o\left(b^{x}\right)$ for every $b>1$ (i.e., for every $b>1$ and every $C>0$ there is a $x_{0}>0$, such that $f(x)<C \cdot b^{x}$, for every $x>x_{0}$ ). That is, given a spanner $A$ of size $c$ and a document $d$ of size $|d| \leq c$, the approximation algorithm returns a tuple $\mathbf{t}$ with $\mathrm{w}_{\mathbf{t}} \geq \frac{\mathrm{opt}}{f(c)}$, where opt is the maximal weight assigned to a tuple $\mathbf{t}$ over $d$ by $A$.

Due to Håstad [Hås01, Theorem 6.5], there is a satisfiable 3CNF formula $\psi$, such that this procedure can at best lead to an $(8 / 7-\varepsilon)$ approximation of the maximal number of satisfiable clauses. Therefore, it follows that

$$
\mathrm{w}_{\mathbf{t}} \leq \frac{\mathrm{opt}}{(8 / 7-\varepsilon)^{k}} .
$$

Let $\mathbf{t} \in \llbracket A_{\psi}^{k} \rrbracket^{\mathbb{K}}\left(d^{k}\right)$ be such an approximation and $\tau_{1}, \ldots, \tau_{k}$ be the corresponding variable assignments of $\psi$. Recall that $\left|A_{\psi}^{k}\right|=c \cdot k$ and $\left|d^{k}\right|=n \cdot k \leq c \cdot k$. Per assumption, there is an approximation algorithm, returning a tuple $\mathbf{t}$ with

$$
\mathrm{w}_{\mathbf{t}} \geq \frac{\mathrm{opt}}{f(c \cdot k)} \text {. }
$$

The tuple $\mathbf{t}$ encodes $k$ variable assignments and the weight of the tuple is the product of the weights of the variable assignments. Let $\tau$ be one of the variable assignments, encoded by $\mathbf{t}$, which satisfy the most clauses. ${ }^{29}$

Thus, combining both inequalities, it must hold that $\frac{\mathrm{opt}}{f(c \cdot k)} \leq \mathrm{w}_{\mathbf{t}} \leq \frac{\mathrm{opt}}{(8 / 7-\varepsilon)^{k}}$. Thus, $(8 / 7-\varepsilon)^{k} \leq f(c \cdot k)$. Recall that the function $f$ is sub-exponential, that is $f(x) \in o\left(b^{x}\right)$ for every $b>1$. Therefore, $(8 / 7-\varepsilon)^{k} \in o\left(b^{(c \cdot k)}\right)$, however, if $1<b<8 / 7-\varepsilon$, this does not hold for arbitrarily large $k$, leading to the desired contradiction.

\section{Enumeration Problems}

In this section we consider computing the output of annotators from the perspective of enumeration problems, where we try to enumerate all tuples with nonzero weight, possibly from large to small. Such problems are highly relevant for (variants of) VSet-automata, as witnessed by the recent literature that explicitly focuses on such automata [ABMN19, $\mathrm{FRU}^{+} 18$ ] or on alternative formalisms [BGJR21, DK21].

An enumeration problem $P$ is a (partial) function that maps each input $i$ to a finite or countably infinite set of outputs for $i$, denoted by $P(i)$. Terminologically, we say that, given $i$, the task is to enumerate $P(i)$.

An enumeration algorithm for $P$ is an algorithm that, given input $i$, writes a sequence of answers to the output such that every answer in $P(i)$ is written precisely once. If $A$ is an enumeration algorithm for an enumeration problem $P$, we say that $A$ runs in preprocessing

\footnotetext{
${ }^{29}$ We note that there might be multiple assignments satisfying the same number of clauses.
} 
$p$ and delay $d$ if the time before writing the first answer is $p(|i|)$, where $|i|$ is the size of the input $i$, and the time between writing every two consecutive answers is $d(|i|)$. By between answers, we mean the number of steps between writing the first symbol from an answer until writing the first symbol of the next answer. We generalize this terminology in the usual way to classes of functions. E.g., an algorithm with linear preprocessing and constant delay has a linear function for $p$ and a constant function for $d$. Notice that we measure with a RAM model with logarithmic word size. Therefore, for a document $d$ and VSet-automaton $A$, an algorithm with a delay that is constant in $|d|$ and polynomial in $|A|$ is able to produce answers that consist of $|A|^{c}$ many words of logarithmic size, for some constant $c$. Thus, such an enumeration algorithm can produce $d$-tuples $\mathbf{t} \in \llbracket A \rrbracket(d)$ as answers.

Given a $\mathbb{K}$-weighted VSet-automaton $A$ and a document $d$, let $f(A, d)$ be the maximal time required for a single addition or multiplication while computing the weight $\llbracket A \rrbracket^{\mathbb{K}}(d)(\mathbf{t})$ for some tuple $\mathbf{t}$. We note that, due to the assumption that $\mathbb{K}$ has an efficient encoding, $f(A, d)$ is at most polynomial in $|A|$ and $|d|$. Furthermore, for instance for finite semirings (like the Boolean semiring or the access control semiring), $f(A, d)$ is constant. If the order of the answers does not matter and the semiring is positive, we can guarantee an enumeration algorithm which has linear preprocessing time and constant delay in the size of the document and polynomial time and delay in the size of $A$ and $f(A, d) .{ }^{30}$ Note that the proof of the theorem essentially requires to go through the entire proof of the main result of Amarilli et al. [ABMN19, Theorem 1.1].

Theorem 8.1. Given a weighted functional VSet-automaton A without $\varepsilon$-transitions over a positive semiring $\mathbb{K}$, and a document $d$, the $\mathbb{K}$-Relation $\llbracket A \rrbracket^{\mathbb{K}}(d)$ can be enumerated with preprocessing linear in $|d|$ and polynomial in $|A|$ and $f(A, d)$, and delay constant in $|d|$ and polynomial in $|A|$ and $f(A, d)$.

Proof Sketch. Amarilli et al. [ABMN19, Theorem 1.1] showed that, given a sequential VSetautomaton $A$ without $\varepsilon$-transitions and a document $d$, one can enumerate $\llbracket A \rrbracket(d)$ with preprocessing time $O\left(\left(|Q|^{\omega+1}+|A|\right) \times|d|\right)$ and with delay $O\left(|V| \times\left(|Q|^{2}+|A| \times|V|^{2}\right)\right)$, where $2 \leq \omega \leq 3$ is an exponent for matrix multiplication, $V$ is the set of variables, and $Q$ the set of states in $A$. In other words, $\llbracket A \rrbracket(d)$ can be enumerated with linear preprocessing and constant delay in $d$, and polynomial preprocessing and delay in $A$. To obtain this result, they view the transition function of $A$ as a (Boolean) transition matrix. Their methods easily extend from the Boolean case to transition matrices over positive semirings. ${ }^{31}$ The claimed complexity for enumeration of the $\mathbb{K}$-Relation $\llbracket A \rrbracket^{\mathbb{K}}(d)$ can be achieved by computing all matrix multiplications over $\mathbb{K}$ instead of $\mathbb{B}$. Furthermore, instead of storing the set $\Lambda$ of current states, one has to store a set of (state,weight)-tuples in order to compute the correct weights of the returned tuples.

Depending on whether or not $f(A, d)$ is constant, we have the following two corollaries.

Corollary 8.2. Given a weighted functional VSet-automaton A over a positive semiring $\mathbb{K}$, and a document $d$, such that $f(A, d)$ is constant. Then the $\mathbb{K}$-Relation $\llbracket A \rrbracket^{\mathbb{K}}(d)$ can be

\footnotetext{
${ }^{30}$ We note that $f(A, d)$ can be polynomial in the size of the document. Thus, strictly speaking, preprocessing (resp., delay) might not be linear (resp., constant) in the size of the document. However, stating the theorem like this enables us to give two direct corollaries (Corollaries 8.2 and 8.3) depending on whether or not $f(A, d)$ is constant.

${ }^{31}$ Note that positivity is required as otherwise weights might sum up or multiply to zero, which may violate the constant delay.
} 
enumerated with preprocessing linear in $|d|$ and polynomial in $|A|$, and delay constant in $|d|$ and polynomial in $|A|$.

Corollary 8.3. Given a weighted functional VSet-automaton A over a positive semiring $\mathbb{K}$, and a document $d$, such that $f(A, d)$ is polynomial in $|A|$ and $|d|$. Then the $\mathbb{K}$-Relation $\llbracket A \rrbracket^{\mathbb{K}}(d)$ can be enumerated with preprocessing linear in $|d|$ and polynomial in $f(A, d)$, and delay constant in $|d|$ and polynomial in $f(A, d)$.

Thus, if the order of the answers does not matter and the semiring is positive, we can guarantee an efficient enumeration algorithm.

We now consider cases in which answers are required to arrive in a certain ordering.

\begin{tabular}{|c|c|}
\hline \multicolumn{2}{|r|}{ Ranked Annotator Enumeration (RA-EnUM) } \\
\hline Given: & $\begin{array}{l}\text { Regular annotator } A \text { over an ordered semiring }(\mathbb{K}, \oplus, \otimes, \overline{0}, \overline{1}) \\
\text { and a document } d \text {. }\end{array}$ \\
\hline Task: & $\begin{array}{l}\text { Enumerate all tuples } \mathbf{t} \in \llbracket A \rrbracket^{\mathbb{K}}(d) \text { in descending order on } \\
\mathbb{K} \text {. }\end{array}$ \\
\hline
\end{tabular}

Theorem 8.4. Let $\mathbb{K}$ be an positively ordered, bipotent semiring, let $A$ be a $\mathbb{K}$-weighted functional VSet-automaton, and let $d \in$ Docs be a document. Then RA-ENUM can be solved with polynomial delay and preprocessing.

Proof. By Proposition 6.7, we can assume that $A$ is an extended functional $\mathbb{K}$-weighted VSet-automaton. Therefore, all runs of $A$ which accept a tuple $\mathbf{t} \in \llbracket A \rrbracket^{\mathbb{K}}(d)$ have the same label. We will use the DAG $G$ we defined in the proof of Theorem 7.5 and run a slight adaptation of Yen's algorithm [Yen71] on $G$.

From the proof of Theorem 7.5 it follows that, given $G$ we can find a path from $s$ to $t$ in $G$ with maximal weight in polynomial time. Let $p=n_{0} \cdot e_{0} \cdot n_{1} \cdots e_{k-1} \cdot n_{k}$ be a path in $G$, where $n_{i} \in N$ and $e_{j} \in E$ for $0 \leq i \leq k$ and $0 \leq j<k$. We denote by $p[i, i]$ the node $n_{i}$, by $p[i, j]$ the path $n_{i} \cdot e_{i} \cdots e_{j-1} \cdot n_{j}$ and by $N(p)$ the set $\left\{n_{i} \mid 0 \leq i \leq k\right\}$ of nodes used by $p$. The Procedure BestWeightEnumeration shows how Yen's algorithm can be adapted for the RA-ENUm problem. Recall that per construction of $D$, all edges which correspond to variable edges of $A$, are labeled by a tuple $(T, i)$, which encodes that the set $T$ is processed after reading $d_{[1, i\rangle}$. Thus, line 12 ensures that, whenever the algorithm reaches line 13, all paths $p[0, i] \cdot p^{\prime}$ where $p^{\prime}$ is a path from $p[i, i]$ to $t$ in $G^{\prime}$ differ from the paths in the set Out in at least one edge label and therefore, no tuple is enumerated multiple times. Observe that the first output of Procedure BestWeightEnumeration is generated after polynomial time. Furthermore, every iteration of the while loop line 4, takes polynomial time. Thus, the algorithm runs with polynomial preprocessing and delay.

\section{Concluding Remarks}

We embarked on a study that incorporates annotation, or weights, in information extraction. We proposed $\mathbb{K}$-annotators as a candidate formalism to study this problem. The $\mathbb{K}$-annotators can be instantiated with weighted VSet-automata, thereby obtaining the regular $\mathbb{K}$-annotators. We showed tha the regular $\mathbb{K}$-annotators have favorable closure properties, such as closure under union, projection, natural join, and, depending on the semiring, also under string selection using regular relations. The first complexity results 


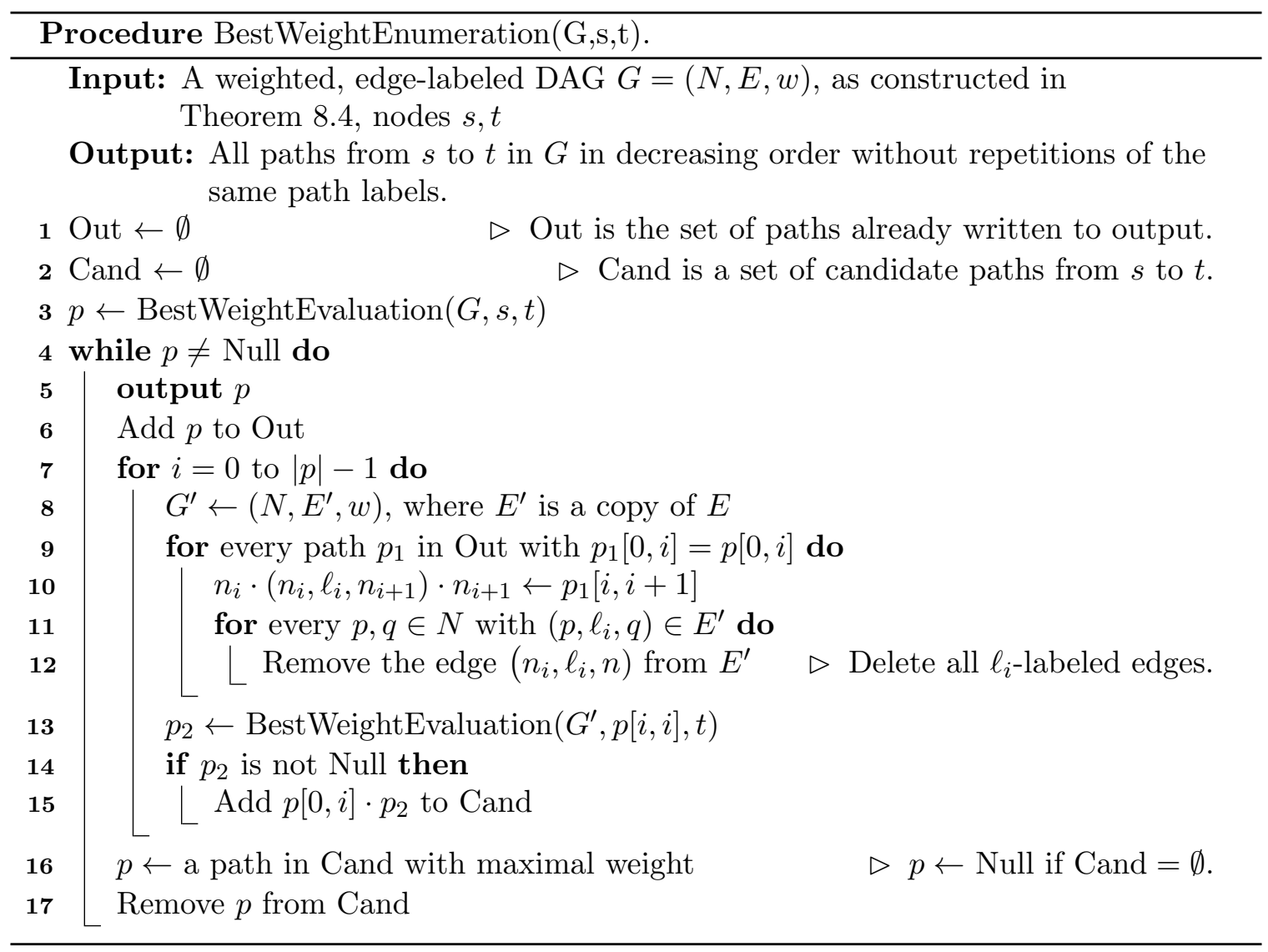

on evaluation problems are encouraging: answer testing is tractable and, depending on the semiring, problems such as the threshold problem, the max tuple problem, and enumeration of answers are tractable too.

We note that the addition of weights to VSet-automata also introduces new challenges. For instance, some questions which are typically studied in database theory are not yet fully understood for weighted automata, which are the basis of weighted VSet-automata. Examples are equivalence and emptiness. Concerning equivalence, it is known that equivalence is undecidable for weighted VSet-automata over the tropical semiring (c.f. Proposition 4.1). In general, however, it is not completely clear for which semirings equivalence is decidable or not. Concerning emptiness, the definition in weighted automata literature is not as database theoreticians would expect. That is, it does not ask if there exists a document $d$ such that the automaton returns at least one tuple with nonzero weight on $d$, but is additionally given a threshold (as in our THRESHOLD problem) and asks if the automaton returns a tuple with at least the threshold weight (which requires an order on the semiring). It is not yet clear how much this threshold influences the complexity of the problem.

An additional challenge is that determinization of weighted automata is a complex matter and not always possible. It is well-known to be possible for the Boolean semiring but, for the tropical semiring (defined as $(\mathbb{Q} \cup\{-\infty\}$, $\max ,+,-\infty, 0)$ ) deterministic weighted automata are strictly less expressive than unambiguous weighted automata, which are strictly less expressive than general weighted automata, cf. Klimann et al. [KLMP04]. 
An interesting direction for future research is to determine the exact connection between our work and past work on Datalog with weights or provenance. As we explained in Section 4.1, one can transform a weighted vset-automaton into a Datalog program over annotated relations according to the semantics of Deutch et al. [DMRT14]. There are other notions of weighted Datalog where the connection to our work appears to be weaker, such as the Independent Choice Logic [Poo95], Prism [SK97] and ProbLog [RKT07] where the existences of ground rules are probabilistically independent events, and the generative Datalog $\left[\mathrm{BtCK}^{+} 17\right.$, GKKL20] where rules are able to produce random numbers. Yet, as far as we know, the publications on weighted versions of Datalog include neither complexity nor closure results that seem to imply the ones of this article. In particular, the tractability of the "provenance circuits" of Deutch et al. [DMRT14] applies to special cases of semirings (e.g., absorptive) that do not arise in our work. Whether the circuit approach can lead to new algorithms or simplification of our results remains to be studied.

\section{ACKNOWLEDGMENT}

The authors are grateful to Matthias Niewerth for many useful discussions and his help regarding Theorem 8.1 and Shaull Almagor for many helpful comments regarding weighted automata. Furthermore, we thank the anonymous reviewers of ICDT 2020 and LMCS for many helpful remarks. Johannes Doleschal is supported by the German Israeli Foundation (GIF), Grant l-1502-407.6/2019. Benny Kimelfeld is supported by the DIP program of the Deutsche Forschungsgemeinschaft (DFG, German Research Foundation), Grant 412400621, and the Israel Science Foundation (ISF), Grant 768/19. Wim Martens is supported by the Deutsche Forschungsgemeinschaft, Grant 431183758. A part of the work of Liat Peterfreund was done while she was affiliated with the Technion, CNRS, IRIF - Université Paris Diderot supported by Fondation Sciences Mathématiques de Paris (FSMP), and University of Edinburgh.

\section{REFERENCES}

[ABK11] Shaull Almagor, Udi Boker, and Orna Kupferman. What's decidable about weighted automata? In International Symposium on Automated Technology for Verification and Analysis (ATVA), pages 482-491, 2011.

[ABMN19] Antoine Amarilli, Pierre Bourhis, Stefan Mengel, and Matthias Niewerth. Constant-delay enumeration for nondeterministic document spanners. In International Conference on Database Theory (ICDT), pages 22:1-22:19, 2019.

[ACJR19] Marcelo Arenas, Luis Alberto Croquevielle, Rajesh Jayaram, and Cristian Riveros. Efficient logspace classes for enumeration, counting, and uniform generation. In ACM Symposium on Principles of Database Systems (PODS), pages 59-73, 2019.

[AH74] Alfred V. Aho and John E. Hopcroft. The Design and Analysis of Computer Algorithms. AddisonWesley Longman Publishing Co., Inc., USA, 1st edition, 1974.

[BGJR21] Pierre Bourhis, Alejandro Grez, Louis Jachiet, and Cristian Riveros. Ranked enumeration of MSO logic on words. In International Conference on Database Theory (ICDT), pages 20:1-20:19, 2021.

$\left[\mathrm{BtCK}^{+} 17\right]$ Vince Bárány, Balder ten Cate, Benny Kimelfeld, Dan Olteanu, and Zografoula Vagena. Declarative probabilistic programming with datalog. ACM Trans. Database Syst., 42(4):22:1-22:35, 2017.

$\left[\mathrm{CKL}^{+} 10\right]$ Laura Chiticariu, Rajasekar Krishnamurthy, Yunyao Li, Sriram Raghavan, Frederick Reiss, and Shivakumar Vaithyanathan. SystemT: An algebraic approach to declarative information 
extraction. In Annual Meeting of the Association for Computational Linguistics (ACL), pages 128-137, 2010.

[CN16] Jason P. C. Chiu and Eric Nichols. Named entity recognition with bidirectional LSTM-CNNs. Trans. Assoc. Comput. Linguistics, 4:357-370, 2016.

[DK09] Manfred Droste and Werner Kuich. Semirings and Formal Power Series, pages 3-28. Springer Berlin Heidelberg, 2009.

[DK21] Shaleen Deep and Paraschos Koutris. Ranked enumeration of conjunctive query results. In International Conference on Database Theory (ICDT), pages 5:1-5:19, 2021.

$\left[\mathrm{DKM}^{+} 19\right]$ Johannes Doleschal, Benny Kimelfeld, Wim Martens, Yoav Nahshon, and Frank Neven. Splitcorrectness in information extraction. In ACM Symposium on Principles of Database Systems (PODS), pages 149-163, 2019.

[DKMP20] Johannes Doleschal, Benny Kimelfeld, Wim Martens, and Liat Peterfreund. Weight annotation in information extraction. In International Conference on Database Theory (ICDT), pages 8:1-8:18, 2020.

[DKV09] Manfred Droste, Werner Kuich, and Heiko Vogler. Handbook of Weighted Automata. Springer, 1st edition, 2009.

[DMRT14] Daniel Deutch, Tova Milo, Sudeepa Roy, and Val Tannen. Circuits for datalog provenance. In International Conference on Database Theory (ICDT), pages 201-212, 2014.

[Eil74] Samuel Eilenberg. Automata, Languages, and Machines. Academic Press, Inc., Orlando, FL, USA, 1974.

[ÉK09] Zoltán Ésik and Werner Kuich. Finite Automata, pages 69-104. Springer Berlin Heidelberg, 2009.

[FGT08] J. Nathan Foster, Todd J. Green, and Val Tannen. Annotated XML: queries and provenance. In ACM Symposium on Principles of Database Systems (PODS), pages 271-280, 2008.

[FKP18] Dominik D. Freydenberger, Benny Kimelfeld, and Liat Peterfreund. Joining extractions of regular expressions. In ACM Symposium on Principles of Database Systems (PODS), pages 137-149, 2018.

[FKRV15] Ronald Fagin, Benny Kimelfeld, Frederick Reiss, and Stijn Vansummeren. Document spanners: A formal approach to information extraction. J. ACM, 62(2):12, 2015.

[FKRV16] Ronald Fagin, Benny Kimelfeld, Frederick Reiss, and Stijn Vansummeren. Declarative cleaning of inconsistencies in information extraction. ACM Trans. Database Syst., 41(1):6:1-6:44, 2016.

[Fre19] Dominik D. Freydenberger. A logic for document spanners. Theory Comput. Syst., 63(7):1679$1754,2019$.

[FRU $\left.{ }^{+} 18\right]$ Fernando Florenzano, Cristian Riveros, Martín Ugarte, Stijn Vansummeren, and Domagoj Vrgoc. Constant delay algorithms for regular document spanners. In ACM Symposium on Principles of Database Systems (PODS), pages 165-177, 2018.

[GKKL20] Martin Grohe, Benjamin Lucien Kaminski, Joost-Pieter Katoen, and Peter Lindner. Generative datalog with continuous distributions. In ACM Symposium on Principles of Database Systems (PODS), pages 347-360, 2020.

[GKT07] Todd J. Green, Gregory Karvounarakis, and Val Tannen. Provenance semirings. In ACM Symposium on Principles of Database Systems (PODS), pages 31-40, 2007.

[Gol99] Jonathan S. Golan. Semirings and their Applications. Springer, Dordrecht, 1999.

[Goo99] Joshua Goodman. Semiring parsing. Computational Linguistics, 25(4):573-605, 1999.

[Hås01] Johan Håstad. Some optimal inapproximability results. J. ACM, 48(4):798-859, July 2001.

[Kir08] Daniel Kirsten. A burnside approach to the termination of Mohri's algorithm for polynomially ambiguous min-plus-automata. RAIRO - Theoretical Informatics and Applications, 42(3):553-581, 2008.

[KLMP04] Ines Klimann, Sylvain Lombardy, Jean Mairesse, and Christophe Prieur. Deciding unambiguity and sequentiality from a finitely ambiguous max-plus automaton. Theoretical Computer Science, 327(3):349 - 373, 2004.

[Kro94] Daniel Krob. The equality problem for rational series with multiplicities in the tropical semiring is undecidable. Int. J. Algebra Comput., 4(3):405-426, 1994.

[LBC04] Yaoyong Li, Kalina Bontcheva, and Hamish Cunningham. SVM based learning system for information extraction. In Deterministic and Statistical Methods in Machine Learning, pages 319-339, 2004. 
[Moh09] Mehryar Mohri. Weighted Automata Algorithms, pages 213-254. Springer Berlin Heidelberg, 2009.

[MRV18] Francisco Maturana, Cristian Riveros, and Domagoj Vrgoc. Document spanners for extracting incomplete information: Expressiveness and complexity. In ACM Symposium on Principles of Database Systems (PODS), pages 125-136, 2018.

$\left[\mathrm{MSV}^{+} 19\right]$ Joshua J. Michalenko, Ameesh Shah, Abhinav Verma, Richard G. Baraniuk, Swarat Chaudhuri, and Ankit B. Patel. Representing formal languages: A comparison between finite automata and recurrent neural networks. In International Conference on Learning Representations (ICLR) Poster, 2019.

[MY18] Franz Mayr and Sergio Yovine. Regular inference on artificial neural networks. In International Cross-Domain Conference on Machine Learning and Knowledge Extraction (CD-MAKE), pages 350-369, 2018.

[PD07] Hoifung Poon and Pedro M. Domingos. Joint inference in information extraction. In $A A A I$ Conference on Artificial Intelligence (AAAI), pages 913-918, 2007.

[PFKK19] Liat Peterfreund, Dominik D. Freydenberger, Benny Kimelfeld, and Markus Kröll. Complexity bounds for relational algebra over document spanners. In ACM Symposium on Principles of Database Systems (PODS), pages 320-334, 2019.

[Poo95] David Poole. Exploiting the rule structure for decision making within the independent choice logic. In Annual Conference on Uncertainty in Artificial Intelligence (UAI), pages 454-463, 1995.

$\left[\mathrm{PPQ}^{+} 17\right]$ Nanyun Peng, Hoifung Poon, Chris Quirk, Kristina Toutanova, and Wen-tau Yih. Cross-sentence n-ary relation extraction with graph LSTMs. Trans. Assoc. Comput. Linguistics, 5:101-115, 2017.

[PtCFK19] Liat Peterfreund, Balder ten Cate, Ronald Fagin, and Benny Kimelfeld. Recursive programs for document spanners. In International Conference on Database Theory (ICDT), volume 127, pages $13: 1-13: 18,2019$.

[Rab63] Michael O. Rabin. Probabilistic automata. Information and Control, 6(3):230 - 245, 1963.

$\left[\mathrm{RBE}^{+} 17\right]$ Alexander Ratner, Stephen H. Bach, Henry R. Ehrenberg, Jason Alan Fries, Sen Wu, and Christopher Ré. Snorkel: Rapid training data creation with weak supervision. Proc. VLDB Endow., 11(3):269-282, 2017.

[RKT07] Luc De Raedt, Angelika Kimmig, and Hannu Toivonen. Problog: A probabilistic prolog and its application in link discovery. In International Joint Conference on Artificial Intelligence (IJCAI), pages 2462-2467, 2007.

[Sak09] Jacques Sakarovitch. Elements of Automata Theory. Cambridge University Press, 2009.

[Sar08] Sunita Sarawagi. Information extraction. Foundations and Trends in Databases, 1(3):261-377, 2008.

[SDNR07] Warren Shen, AnHai Doan, Jeffrey F. Naughton, and Raghu Ramakrishnan. Declarative information extraction using Datalog with embedded extraction predicates. In VLDB, pages 1033-1044, 2007.

[Seg06] Roberto Segala. Probability and nondeterminism in operational models of concurrency. In International Conference on Concurrency Theory (CONCUR), pages 64-78, 2006.

[SK97] Taisuke Sato and Yoshitaka Kameya. PRISM: A language for symbolic-statistical modeling. In International Joint Conference on Artificial Intelligence (IJCAI), pages 1330-1339, 1997.

[SM12] Charles A. Sutton and Andrew McCallum. An introduction to conditional random fields. Foundations and Trends in Machine Learning, 4(4):267-373, 2012.

[SS21] Markus L. Schmid and Nicole Schweikardt. A purely regular approach to non-regular core spanners. In International Conference on Database Theory (ICDT), pages 4:1-4:19, 2021.

[STS18] Roy Schwartz, Sam Thomson, and Noah A. Smith. Bridging CNNs, RNNs, and weighted finitestate machines. In Annual Meeting of the Association for Computational Linguistics (ACL), pages 295-305, 2018.

$\left[\mathrm{SWW}^{+} 15\right]$ Jaeho Shin, Sen Wu, Feiran Wang, Christopher De Sa, Ce Zhang, and Christopher Ré. Incremental knowledge base construction using DeepDive. Proc. VLDB Endow., 8(11):1310-1321, 2015.

[TSZB03] David Torrents, Mikita Suyama, Evgeny Zdobnov, and Peer Bork. A genome-wide survey of human pseudogenes. Genome research, 13(12):2559-2567, 2003. 
[WGY18] Gail Weiss, Yoav Goldberg, and Eran Yahav. Extracting automata from recurrent neural networks using queries and counterexamples. In International Conference on Machine Learning (ICML), volume 80, pages 5244-5253, 2018.

[WJ94] Lusheng Wang and Tao Jiang. On the complexity of multiple sequence alignment. Journal of Computational Biology, 1(4):337-348, 1994.

[Yen71] Jin Y. Yen. Finding the $k$ shortest loopless paths in a network. Management Science, 17(11):712716, 1971. URL: http://www.jstor.org/stable/2629312. 